\title{
Multiscale modelling of mesoscopic phenomena triggered by quantum events: light-driven azo-materials and beyond $\dagger$
}

\author{
Marcus Böckmann, ${ }^{a}$ Dominik Marx,${ }^{a}$ Christine Peter ${ }^{b}$ Luigi Delle Site, ${ }^{b}$ \\ Kurt Kremer $^{b}$ and Nikos L. Doltsinis*c
}

\begin{abstract}
The macroscopic functionality of soft (bio-)materials is often triggered by quantum-mechanical events which are highly local in space and time. In order to arrive at the resulting macroscopically observable phenomena, many orders of magnitude need to be bridged on both the time and the length scale. In the present paper, we first introduce a range of simulation methods at different scales as well as theoretical approaches to form bridges between them. We then outline a strategy to develop an adaptive multiscale simulation approach which connects the quantum to the mesoscopic level by bringing together ab initio molecular dynamics (QM), classical (force field) molecular dynamics (MM), and coarse grained (CG) simulation techniques. With a multitude of photoactive materials in mind, we apply our methodology to a prototypical test case - light-induced phase transitions in a liquid crystal containing the azobenzene photoswitch.
\end{abstract}

\section{Introduction}

Macroscopically observable phenomena typically involve a number of interconnected processes on different length and time scales, the triggering event often being a specific quantum-mechanical process local in time and space. Simulation of mesoscopic or even macroscopic phenomena therefore requires the use of different hierarchical levels of theory. Traditional simulation methods have specialized on specific time and length scales without connecting to the levels above or below.

A first principles - quantum-mechanical (QM) - dynamical treatment of soft matter can be achieved by employing the $a b$ initio molecular dynamics (AIMD) method. ${ }^{1-3}$ This approach takes into account the system's electronic structure by solving the Schrödinger equation "on the fly" in an approximate fashion within the framework of density functional theory. Applications, however, are limited to systems containing a few hundred atoms and to trajectories of a few tens of picoseconds, due to the computational cost involved.

The next level up from the QM description is the molecular mechanics (MM) approach, i.e. classical molecular dynamics using (empirical) force field potentials. This method can be

\footnotetext{
${ }^{a}$ Lehrstuhl für Theoretische Chemie, Ruhr-Universität Bochum, 44780 Bochum, Germany

${ }^{b}$ Max-Planck Institut für Polymerforschung, Ackermannweg 10,

55128 Mainz, Germany

'Department of Physics, King's College London, Strand,

London WC2R 2LS,UK.E-mail:nikos.doltsinis@kcl.ac.uk

$\dagger$ This article was submitted following the 1st workshop on Energy

Materials, organised by The Thomas Young Centre, and held on 7-9

September 2010 at University College London.
}

applied to systems of a few thousand atoms on the nanosecond time scale. However, the possibility of chemical reactions, i.e. bond breaking and formation, is typically not accounted for. Similarly, inherently quantum-mechanical events such as photoinduced processes cannot be described. For this reason, hybrid QM/MM techniques have been developed, which treat the chemically active centre quantum-mechanically and the chemically inert environment classically. ${ }^{4-9}$

Going to larger time and length scales requires abandonment of the atomistic resolution. The number of interactions in a system can be significantly reduced if a number of atomic centres are mapped onto an effective particle. ${ }^{10,11}$ Such coarse grained (CG) models have been used successfully to study polymeric systems, ${ }^{12}$ such as BPA-PC ${ }^{13,14}$ or polystyrene, ${ }^{15-20}$ as well as lipid systems, ${ }^{21-24}$ peptides,${ }^{25-29}$ proteins $^{30-32}$ and many more. A fairly extensive overview of recent research can be found in the Faraday discussions FD $144^{33}$ on multiscale simulation in soft matter.

Coarse grained interaction potentials are typically derived from the atomistic model by reproducing thermodynamic and/ or structural properties. ${ }^{22,34-41}$ To be able to switch back from the $\mathrm{CG}$ to $\mathrm{MM}$ description whenever a higher resolution is required, suitable back-mapping recipes have been developed. ${ }^{13,15,27-29,42-45}$

Until recently, attempts to connect more than two hierarchical levels had been very rare. The problem of interlinking the three simulation levels QM, MM, and CG was first tackled, for molecular systems, by this consortium. ${ }^{46}$ An additional level of complexity arose from our ambition to go beyond the standard QM description, i.e. a ground state Born-Oppenheimer treatment, and use a nonadiabatic approach (na-QM) coupling multiple 
electronic states. This is necessary to model, for instance, lightinduced processes in biosystems or photoswitchable materials.

In the present paper, we describe our strategy for developing a na-QM/MM/CG multiscale simulation approach applicable to a wide range of mesoscopic phenomena triggered by the absorption of a photon by a chromophore. As an application prototypical of the vast area of light-controllable materials based on the azobenzene (AB) photoswitch, ${ }^{47-58}$ we chose the photoinduced phase transitions in the $8 \mathrm{AB} 8$ liquid crystal.

We approached our target of developing a na-QM/MM/CG multiscale method from two angles. Coming from the quantummechanical level, we coupled a previously developed na-QM approach $^{59,60}$ to the MM level using a suitable QM/MM interface. ${ }^{61}$ The new na-QM/MM simulation tool is applicable to a wide range of (photoexcited) systems and has been used to study the photoisomerisation of $\mathrm{AB}$ in the bulk liquid phase ${ }^{62,63}$ As a prerequisite, a force field suitable for modelling photoswitchable materials based on AB-containing organic chains has been developed ${ }^{46}$ by mapping the results from QM (AIMD) simulations. A CG model of the 8AB8 liquid crystal has also been derived based on the atomistic azo force field. ${ }^{44}$

The necessary tools are now in place to perform multiscale simulations of photoactive azo-materials combining the na-QM, MM, and CG hierarchical levels. Such simulations can be carried out in two different ways. The first, sequential approach involves separate simulations at the different levels. The cross-linking will be achieved by passing on the information obtained from the simulation at the next higher/lower level in the shape of initial conditions (see Fig. 1a). This procedure has the advantage that the overall timespan covered by the simulation is long, as it is essentially determined by the CG level. On the other hand, the effects of a larger CG environment on the QM subsystem cannot be taken into account. In the second approach, simultaneous na-QM/MM/CG simulations are performed (Fig. 1b). This has not yet been achieved and
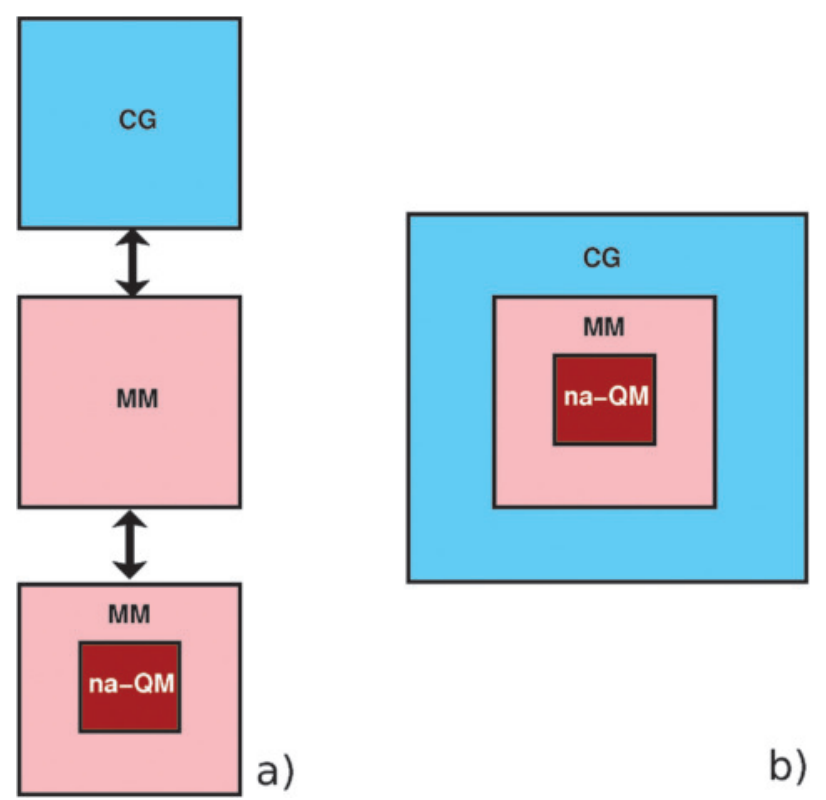

Fig. 1 Schematic representations of the two different multiscale simulation approaches: sequential (a) and simultaneous (b). requires the development of a new computer code featuring the two interfaces, $\mathrm{QM} / \mathrm{MM}$ and $\mathrm{MM} / \mathrm{CG}$, discussed above. This simultaneous method allows the simulation of larger systems compared to the sequential approach. However, the overall timescale is determined by the fast QM process.

Another important issue is the partitioning of the total system into the QM, MM, and CG subsystems. In standard $\mathrm{QM} / \mathrm{MM}$ simulations, for instance, the partitioning is fixed, i.e. atoms initially assigned to the $\mathrm{QM}$ and $\mathrm{MM}$ regions remain in their respective partitions throughout the simulation. Exchange of particles between the QM and MM subsystems, caused either by a moving QM/MM boundary or by particle diffusion through the $\mathrm{QM} / \mathrm{MM}$ boundary, is typically not taken into account. However, these are key issues, for instance, in the simulation of crack propagation in materials and solvated active sites in biomolecules. Very few adaptive $\mathrm{QM} / \mathrm{MM}$ resolution schemes have so far been proposed. ${ }^{64-70}$ They generally suffer from an excessive computational overhead, and, those explicitly treating electrons, lack conceptual consistency (see Section II E), hence the number of applications is small.

In addition to a changing QM subsystem, it is often of great importance to study the interaction between two or more QM centres at a given time. In photoactive materials, this situation occurs when two chromophores in close proximity are excited within a short time span. To what extent is the final state (cis or trans) of one $\mathrm{AB}$ chromophore dependent on the photoisomerisation dynamics of the other? There is no $\mathrm{QM} / \mathrm{MM}$ scheme at present that allows for two separate QM regions. Furthermore, in a real system different molecular centres may be activated at different times, e.g. different azobenzene chromophores absorb photons and undergo photoisomerisation. This scenario requires "switching off" one QM subsystem (i.e. turning it into an MM system) and simultaneously "switching on" another QM subsystem using a suitable switching function.

At the mesoscopic level, in analogy to the QM/MM case, certain physical problems call for a hybrid $\mathrm{MM} / \mathrm{CG}$ approach where the same partitioning problems persist. $\mathrm{MM} / \mathrm{CG}$ dual resolution methods have been developed recently in the field of materials science. A novel adaptive resolution molecular dynamics technique has been proposed as part of the current project ${ }^{71-74}$ in which a molecule's level of description (i.e. MM or CG) is dynamically adjusted according to its position relative to the $\mathrm{MM} / \mathrm{CG}$ boundary.

In Sections II A to II E we present a brief synopsis of the different hierarchical molecular dynamics simulation methods and the specific developments carried out by this consortium including a nonadiabatic QM/MM method and adaptive partitioning schemes. In Sections III A to III $\mathrm{C}$ we then discuss applications of these techniques to various aspects contributing to the ultimate goal of this study, namely a multiscale simulation study of the light-induced phase transition in the 8AB8 liquid crystal. We start with QM simulations of a single $\mathrm{AB}$ molecule in the gas phase. As a reference for the subsequent condensed phase simulations, we investigate the photoisomerisation of isolated $\mathrm{AB}$ using our na-QM method. Based on the dynamical QM data a classical atomistic force field is developed, which is then employed in 
na-QM/MM and MM simulations of liquid AB. Analogously, an extended atomistic force field is derived for the $\mathrm{AB}$ derivative 8AB8 from $\mathrm{QM}$ simulations. MM simulations of 8AB8 serve as a reference for the parametrisation of a $\mathrm{CG}$ force field for $8 \mathrm{AB} 8$.

\section{Methodological approach and developments}

\section{A. Ab initio molecular dynamics}

1. Born-Oppenheimer molecular dynamics. In the so-called Born-Oppenheimer implementation of AIMD ${ }^{2}$ the nuclei are propagated by integration of Newton's equation,

$$
-\nabla_{I} \mathrm{E}_{k}=M_{I} \ddot{R}_{I}
$$

where $M_{I}$ and $\boldsymbol{R}_{I}$ are the mass and position of nucleus $I$. The exact energy $E_{k}$ is replaced with the eigenvalue, $\tilde{E}_{k}$, of some approximate electronic Hamiltonian, $\tilde{\mathscr{H}}_{\text {el }}$, which is calculated by iterative wavefunction optimization at each time step. For the electronic ground state, i.e. $k=0$, the use of Kohn-Sham (KS) density functional theory ${ }^{75,76}$ has become increasingly popular.

2. Car-Parrinello molecular dynamics. In order to further increase computational efficiency, Car and Parrinello introduced a technique to bypass the need for wavefunction optimization at each molecular dynamics step. ${ }^{1,2}$ Instead, the molecular wavefunction, $\Phi_{k}$, is dynamically propagated along with the atomic nuclei according to the equations of motion

$$
\begin{aligned}
M_{I} \ddot{\boldsymbol{R}}_{I} & =-\nabla_{I}\left\langle\Phi_{k}\left|\tilde{\mathscr{H}}_{\mathrm{el}}\right| \Phi_{k}\right\rangle \\
\mu_{i} \ddot{\phi}_{i} & =-\frac{\delta}{\delta \phi_{i}^{\star}}\left\langle\Phi_{k}\left|\tilde{\mathscr{H}}_{\mathrm{el}}\right| \Phi_{k}\right\rangle+\sum_{j} \lambda_{i j} \phi_{j}
\end{aligned}
$$

where the KS one-electron orbitals $\phi_{i}$ are kept orthonormal by the Lagrange multipliers $\lambda_{i j}$; the fictitious orbital mass $\mu_{i}$ is chosen such as to separate adiabatically electronic and nuclear degrees of freedom. These are the Euler-Lagrange equations

$$
\frac{\mathrm{d}}{\mathrm{d} t} \frac{\partial \mathscr{L}}{\partial \dot{q}}=\frac{\partial \mathscr{L}}{\partial q},\left(q=\boldsymbol{R}_{I}, \phi_{i}^{\star}\right)
$$

for the Car-Parrinello Lagrangian ${ }^{1}$

$$
\begin{aligned}
\mathscr{L}= & \sum_{I} \frac{1}{2} M_{I} \dot{\boldsymbol{R}}_{I}^{2}+\sum_{i} \frac{1}{2} \mu_{i}\left\langle\dot{\phi}_{i} \mid \dot{\phi}_{i}\right\rangle \\
& -\left\langle\Phi_{k}\left|\tilde{\mathscr{H}}_{\mathrm{el}}\right| \Phi_{k}\right\rangle+\sum_{i j} \lambda_{i j}\left(\left\langle\phi_{i} \mid \phi_{j}\right\rangle-\delta_{i j}\right)
\end{aligned}
$$

that is formulated here for an arbitrary electronic state $\Phi_{k}$, an arbitrary electronic Hamiltonian $\tilde{\mathscr{H}}_{\mathrm{el}}$, and an arbitrary basis (i.e. without invoking the Hellmann-Feynman theorem).

3. Ab initio molecular dynamics beyond the Born-Openheimer approximation. A detailed description of the nonadiabatic AIMD surface hopping method has been published elsewhere, ${ }^{59,60,77,78}$ it shall only be summarized briefly here. We have adopted a mixed quantum-classical picture treating the atomic nuclei according to classical mechanics and the electrons quantum-mechanically. In our two-state model, the total electronic wavefunction, $\Psi$, is represented as a linear combination of the $S_{0}$ and $S_{1}$ adiabatic state functions, $\Phi_{0}$ and $\Phi_{1}$,

$$
\Psi(\boldsymbol{r}, t)=a_{0}(t) \Phi_{0}(\boldsymbol{r}, \boldsymbol{R})+a_{1}(t) \Phi_{1}(\boldsymbol{r}, \boldsymbol{R})
$$

where the time-dependent expansion coefficients $a_{0}(t)$ and $a_{1}(t)$ are to be determined such that $\Psi$ is a solution to the time-dependent electronic Schrödinger equation,

$$
\mathscr{H}(r, \boldsymbol{R}(t)) \Psi(\mathbf{r}, t)=i \hbar \frac{\partial}{\partial t} \Psi(\mathbf{r}, t)
$$

$\boldsymbol{r}$ being the electronic position vector, $\boldsymbol{R}(t)$ the nuclear trajectory.

In the present case, our adiabatic basis functions are the $S_{0}$ closed-shell Kohn-Sham ground state determinant,

$$
\Phi_{0}=\left|\phi_{1}^{(0)} \bar{\phi}_{1}^{(0)} \cdots \phi_{n}^{(0)} \bar{\phi}_{n}^{(0)}\right\rangle
$$

and the orthonormalized $S_{1}$ wavefunction

$$
\Phi_{1}=\frac{1}{\sqrt{1-S^{2}}}\left[-S \Phi_{0}+\Phi_{1}^{\prime}\right]
$$

where

$$
S=\left\langle\Phi_{0} \mid \Phi_{1}^{\prime}\right\rangle
$$

is the overlap between the ground state wavefunction and the ROKS excited state wavefunction ${ }^{79-81}$

$$
\Phi_{1}^{\prime}=\frac{1}{\sqrt{2}}\left\{\left|\phi_{1}^{(1)} \bar{\phi}_{1}^{(1)} \cdots \phi_{n}^{(1)} \bar{\phi}_{n+1}^{(1)}\right\rangle+\left|\phi_{1}^{(1)} \bar{\phi}_{1}^{(1)} \cdots \bar{\phi}_{n}^{(1)} \phi_{n+1}^{(1)}\right\rangle\right\}
$$

$n$ being half the (even) number of electrons. Separate variational optimization of $\Phi_{0}$ and $\Phi_{1}^{\prime}$ generally results in nonorthogonality, the molecular orbitals $\phi_{l}^{(0)}$ and $\phi_{l}^{(0)}$ are different. Please note, however, that for small $S, \Phi_{1} \approx \Phi_{1}^{\prime}$.

Substitution of ansatz (6) into (7) and integration over the electronic coordinates following multiplication by $\Phi_{k}^{*}(k=0,1)$ from the left yields the coupled equations of motion for the wavefunction coefficients

$$
\dot{a}_{k}(t)=-\frac{i}{\hbar} a_{k}(t) E_{k}-\sum_{l} a_{l}(t) D_{k l}(k, l=0,1)
$$

where $E_{k}$ is the energy eigenvalue associated with the wavefunction $\Phi_{k}$. For the nonadiabatic coupling matrix elements

$$
D_{k l}=\left\langle\Phi_{k}\left|\frac{\partial}{\partial t}\right| \Phi_{l}\right\rangle
$$

the relations $D_{k k}=0$ and $D_{k l}=-D_{l k}$ hold, as our $\Phi_{k}$ are real and orthonormal.

In the Car-Parrinello molecular dynamics (CP-MD) formalism $^{1,2}$ (see Section II A 2), computation of the nonadiabatic coupling elements, $D_{k l}$, is straightforward and efficient, since the orbital velocities, $\dot{\phi}_{l}$, are available at no additional cost due to the underlying dynamical propagation scheme. If, instead of being dynamically propagated, the wavefunctions are optimized at each point of the trajectory (so-called Born-Oppenheimer mode, see Section II A 1), the nonadiabatic coupling elements are calculated using a finite difference scheme. 
Numerical integration of eqn (12) yields the expansion coefficients $a_{k}$, whose square moduli, $\left|a_{0}\right|^{2}$ and $\left|a_{1}\right|^{2}$, can be interpreted as the occupation numbers of ground and excited states, respectively.

Following Tully's fewest switches criterion $^{82}$ recipe, the nonadiabatic transition probability from state $k$ to state $l$ is

$$
\Pi_{k l}=\max \left(0, P_{k l}\right)
$$

with the transition parameter

$$
P_{k l}=-\delta t \frac{\frac{d}{d t}\left|a_{k}\right|^{2}}{\left|a_{k}\right|^{2}}
$$

where $\delta t$ is the MD time step.

A hop from surface $k$ to surface $l$ is carried out when a uniform random number $\zeta<\Pi_{k l}$ provided that the potential energy $E_{l}$ is smaller than the total energy of the system. The latter condition rules out any so-called classically forbidden transitions. After each surface jump atomic velocities are rescaled in order to conserve total energy. In the case of a classically forbidden transition, we retain the nuclear velocities, since this procedure has been demonstrated to be more accurate than alternative suggestions. ${ }^{83}$

The two-state surface hopping formalism presented here can be easily generalized to include multiple excited states. ${ }^{82}$ However, calculating a large number of electronic states including nonadiabatic couplings between them from first principles is often either not straightforward or too computationally demanding in practice. Our two-state approach can present a severe limitation in cases where at least three electronic states are required to capture the system's chemistry or physics.

\section{B. Non-Born-Oppenheimer QM/MM molecular dynamics}

To overcome the size limitations of the nonadiabatic AIMD approach presented in the previous section, the method has been extended and implemented in a QM/MM framework, in which the electrons in the QM subsystem are described by a total wavefunction $\Psi^{\mathrm{QM} / \mathrm{MM}}$ satisfying the time-dependent Schrödinger equation (TDSE), whereas the photochemically inert environment is described by an analytical force field. The $\mathrm{QM} / \mathrm{MM}$ coupling is established via a Hamiltonian $^{61} \mathscr{H}^{\mathrm{QM} / \mathrm{MM}}$ which is a function of all the nuclear coordinates, i.e. both of the QM and the $\mathrm{MM}$ subsystems. Likewise, the total wavefunction, $\Phi^{\mathrm{QM} / \mathrm{MM}}$, depends on the entire set of nuclear coordinates and is expanded

$$
\Psi^{\mathrm{QM} / \mathrm{MM}}(\boldsymbol{r}, \boldsymbol{R}, t)=\sum_{i} a_{i}(t) \Phi_{i}^{\mathrm{QM} / \mathrm{MM}}(\boldsymbol{r}, \boldsymbol{R})
$$

in terms of known electronic state functions, $\Phi_{i}^{\mathrm{QM} / \mathrm{MM}}(\boldsymbol{r}, \boldsymbol{R})$. The time-dependent expansion coefficients, $a_{i}(t)$, are determined by inserting this ansatz into the TDSE, resulting in a system of coupled differential equations,

$$
\dot{a}_{i}(t)=-\frac{i}{\hbar} a_{i}(t) E_{i}^{\mathrm{QM} / \mathrm{MM}}-\sum_{j} a_{j}(t) C_{i j}^{\mathrm{QM} / \mathrm{MM}}
$$

where $E_{i}^{\mathrm{QM} / \mathrm{MM}}$ is the energy of electronic state $i$, and

$$
\begin{aligned}
C_{i j}^{\mathrm{QM} / \mathrm{MM}} & =\left\langle\Phi_{i}^{\mathrm{QM} / \mathrm{MM}}\left|\frac{\partial}{\partial t}\right| \Phi_{j}^{\mathrm{QM} / \mathrm{MM}}\right\rangle \\
& =\dot{\mathbf{R}}\left\langle\Phi_{i}^{\mathrm{QM} / \mathrm{MM}}\left|\frac{\partial}{\partial \mathbf{R}}\right| \Phi_{j}^{\mathrm{QM} / \mathrm{MM}}\right\rangle
\end{aligned}
$$

are the nonadiabatic couplings between states $i$ and $j$.

The present two-state implementation couples nonadiabatically the closed-shell Kohn-Sham ground state, $\Phi_{0}^{\mathrm{QM} / \mathrm{MM}}$, to the re-orthonormalized restricted open-shell Kohn-Sham (ROKS) representation ${ }^{79,84}$ of the $S_{1}$ first singlet excited state, $\Phi_{1}^{\mathrm{QM} / \mathrm{MM}}$, following the successful single-scale na-QM technique. ${ }^{59,85-90}$ As a two-determinant representation (for reviews see ref. 2,3,60), the ROKS $S_{1}$ state provides an improved reference to compute nonadiabatic couplings, ${ }^{91}$ and yields reliable $S_{1}$ nonradiative lifetimes and decay mechanisms when nonadiabatically coupled to the KS ground state; ${ }^{59,85-90}$ the level of accuracy obtained by this efficient approach for the system of interest to this study, i.e. AB photoisomerisation, will be demonstrated in detail in Section III A 2a.

In addition to this QM component, a force field parameterization suitable for condensed phase simulations ${ }^{46}$ must be used to define the $\mathrm{MM}$ part of $\mathscr{H}^{\mathrm{QM} / \mathrm{MM}}$. Importantly, the consistent electron density in the excited state, which deviates from that of the ground state, has to be used to include the $\mathrm{QM} \leftrightarrow \mathrm{MM}$ electrostatic coupling within $\mathscr{H}^{\mathrm{QM} / \mathrm{MM}}$.

\section{Classical atomistic molecular dynamics}

Generally, an empirical force-field consists of terms that model the non-bonded interactions $\left(E_{\text {nonbond }}\right)$, which include both the van der Waals and Coulombic interactions, the bond interactions $\left(E_{\text {bond }}\right)$, the angle bending interactions $\left(E_{\text {angle }}\right)$ and the dihedral (bond rotations) interactions ( $\left.E_{\text {dihedral }}\right)$ :

$$
E(\mathbf{R})=E_{\text {nonbond }}+E_{\text {bond }}+E_{\text {angle }}+E_{\text {dihedral }} .
$$

From the large number of force field flavours we chose the Gromos force field ${ }^{92}$ in which covalent bonds are described by

$$
\begin{gathered}
E_{\text {bond }}=\sum_{i}^{N_{b}} \frac{1}{4} k_{b, i}\left(R_{i}^{2}-R_{0, i}^{2}\right)^{2} \\
E_{\text {angle }}=\sum_{i}^{N_{\theta}} \frac{1}{2} k_{\theta, i}\left(\cos \theta_{i}-\cos \theta_{0, i}\right)^{2} \\
E_{\text {dihedral }}=E_{\text {dih,improp }}+E_{\text {dih,prop }}
\end{gathered}
$$

where

$$
E_{\text {dih,improp }}=\sum_{i}^{N_{\xi}} \frac{1}{2} k_{\xi, i}\left(\xi_{i}-\xi_{0, i}\right)^{2}
$$

and

$$
E_{\text {dih,prop }}=\sum_{i}^{N_{\phi}} k_{\phi, i}\left[1+\cos \left(\delta_{i}\right) \cos \left(m_{i} \phi_{i}\right)\right]
$$

The nonbonded interactions are given by

$$
E_{\text {nonbond }}=E_{\mathrm{vdW}}+E_{\mathrm{el}}
$$


where

and

$$
E_{\mathrm{vdW}}=\sum_{i}^{N_{p}} \frac{C_{12, i}}{R_{i}^{12}}-\frac{C_{6, i}}{R_{i}^{6}}
$$

$$
E_{\mathrm{el}}=\sum_{i}^{N_{p}} \frac{Q_{i}}{4 \pi \varepsilon_{0} R_{i}}
$$

$Q_{i}$ being the product of the two atomic charges involved in pair $i$.

\section{Coarse grained classical molecular dynamics}

Many different approaches have been followed to systematically build up CG models in such a way that they can be used in a multiscale simulation framework where one wants to switch back and forth between different levels of resolution. The main objective of this type of coarse graining efforts is to reproduce structural as well as thermodynamic properties of a higher resolution model. That means the $\mathrm{CG}$ model should sample phase space consistently with the atomistic system (projected onto coarse degrees of freedom). There are various routes to approach this problem: on the one hand there are coarse graining methods where the derivation of interaction potentials between CG particles is targeted at thermodynamic properties such as energies or free energies, for example partitioning data. ${ }^{22,37}$ While this approach is particularly well suited to reproduce processes where for example hydrophilicity/hydrophobicity arguments play a decisive role it does not per se guarantee reproduction of the structure of the system. ${ }^{93}$ On the other hand there are so-called structurebased methods, where the CG interactions are chosen such that the model reproduces certain structure properties - often described by a set of radial distribution functions obtained from all-atom molecular simulations. ${ }^{34,35,41,44}$ While these structure-based methods by construction reproduce local structures and are thus well suited to reinsert atomistic coordinates, it is not a priori clear to which extent these models reproduce thermodynamic properties of the system. It is also not a priori clear how well they reproduce higher-order (e.g. three-body) structural correlations if those had not been included in the parameterization process. ${ }^{94}$ In CG models derived by the so-called force matching (or a related) method $^{95-97}$ the CG force field is determined such that the difference between the (instantaneous) $\mathrm{CG}$ forces and the forces in the underlying atomistic system is minimized. It can be shown that this corresponds to an optimization of the CG interactions to reproduce a many-body multidimensional potential of mean force of the underlying atomistic system. Thus the method is related to the above structurebased CG methods, with the difference that those usually rely on pair distribution functions, i.e. pair potentials of mean force. $^{29,38,94,98}$ Note that there is currently intensive research being carried out to investigate the limitations of the different approaches and whether-and if yes how-it is possible to derive coarse grained potentials that are both thermodynamically as well as structurally consistent with an atomistic description, ${ }^{36,99}$ transferable to different state points or system compositions, ${ }^{39,98,100}$ etc.
In the following we will describe the structure-based method used to parameterize the CG model for the liquid crystalline 8AB8 system: ${ }^{44}$ in many $\mathrm{CG}$ approaches the total set of $\mathrm{CG}$ interaction functions is separated into bonded/covalent and nonbonded potentials which are developed separately. This approach relies on the assumption that the total potential energy $U^{\mathrm{CG}}$ can be separated into the respective contributions

$$
U^{\mathrm{CG}}=\sum U_{\mathrm{B}}^{\mathrm{CG}}+\sum U_{\mathrm{NB}}^{\mathrm{CG}} .
$$

Bonded interactions are derived such that the conformational statistics of the molecules is represented correctly in the CG model. These conformational distributions $P^{\mathrm{CG}}$ are usually characterized by specific bond lengths $R$, angles $\theta$, and torsions $\phi$ between any pair, triple and quadruple of $C G$ beads respectively, i.e. $P^{\mathrm{CG}}(R, \theta, \phi, T)$. The target distributions are determined by sampling the conformational degrees of freedom at atomistic resolution (the different sampling methods will be discussed in more detail below). If one assumes that the different $\mathrm{CG}$ internal degrees of freedom are uncorrelated, $P^{\mathrm{CG}}(R, \theta, \phi, T)$ factorizes into independent probability distributions of bond, angle and torsional degrees of freedom

$$
P^{\mathrm{CG}}(R, \theta, \phi, T)=P^{\mathrm{CG}}(R, T) P^{\mathrm{CG}}(\theta, T) P^{\mathrm{CG}}(\phi, T) .
$$

The individual probability distributions $P^{\mathrm{CG}}(R, T)$, $P^{\mathrm{CG}}(\theta, T)$, and $P^{\mathrm{CG}}(\phi, T)$ are then Boltzmann inverted to obtain the corresponding potentials:

$$
\begin{aligned}
U^{\mathrm{CG}}(R, T) & =-k_{\mathrm{B}} T \ln \left(P^{\mathrm{CG}}(R, T) / R^{2}\right)+C_{R} \\
U^{\mathrm{CG}}(\theta, T) & =-k_{\mathrm{B}} T \ln \left(P^{\mathrm{CG}}(\theta, T) / \sin (\theta)\right)+C_{\theta} \\
U^{\mathrm{CG}}(\phi, T) & =-k_{\mathrm{B}} T \ln P^{\mathrm{CG}}(\phi, T)+C_{\phi},
\end{aligned}
$$

with $C_{R}, C_{\theta}$, and $C_{\phi}$ being irrelevant constants used to set the minima of the respective potentials to zero. In many cases however this factorization leads to artifacts in the CG model. Partially this can be improved by a better choice of coarse units (mapping points). In addition intramolecular potentials can be introduced to take care of this problem. ${ }^{20}$

In structure-based approaches to derive nonbonded interaction functions for coarse grained models, the inverse Monte Carlo or the iterative Boltzmann inversion method ${ }^{34,101}$ can be used to numerically generate a tabulated potential that precisely reproduces a given radial distribution function $g(R)$. These methods rely on an initial guess for a nonbonded potential $U_{\mathrm{NB}, 0}^{\mathrm{CG}}$. Often the Boltzmann inverse of the target $g(R)$, i.e. the potential of mean force,

$$
U_{\mathrm{NB}, 0}^{\mathrm{CG}}=-k_{\mathrm{B}} T \ln g(R),
$$

is used, with which one then performs a coarse grained simulation of the liquid. The resulting structure of this first step will not match the target structure as the potential of mean force is - due to multibody interactions - only in the limit of very high dilution a good estimate for the potential energy. The iterative Boltzmann method uses the following iteration scheme

$$
U_{\mathrm{NB}, i+1}^{\mathrm{CG}}=U_{\mathrm{NB}, i}^{\mathrm{CG}}+k_{B} T \ln \left(\frac{g_{i}(R)}{g(R)}\right),
$$


with which the original guess can be self-consistently refined until the desired structure is obtained. The other option would be to stick to the more complex coupled potentials. The recently developed VOTCA package ${ }^{94}$ offers an automated way to determine $U^{\mathrm{CG}}$ once the mapping is defined.

\section{E. Adaptive resolution molecular dynamics}

In the previous section we have discussed how atomistic-based coarse-graining strategies allow us to reduce the number of degrees of freedom of a problem in order to study properties which are typical of mesoscopic (macroscopic) scales where atomistic resolution does not play a direct role. However in many situations occurring in soft matter there is not a real separation of scales, rather there is a delicate interconnection between them and this then defines the properties of the system. This means, for example, that given a system there may exist regions of space where something of particular interest happens at the atomistic level while in the rest of the system a coarse-grained resolution is sufficient to describe its equilibrium thermodynamic properties. In such cases one must be able to properly couple the detailed description of the atomistic scale in the interesting region with the larger scale coarse-grained description of the rest of the system. For liquids and soft matter, which often are characterized by large fluctuations, the coupling must occur in a way that there is free exchange of particles between the two regions. A molecule that crosses the boundary of the atomistic region slowly loses the atomistic degrees of freedom and becomes a coarse-grained molecule, and vice versa from the atomistic to the coarsegrained region. This adaptive resolution process should be such that the overall thermodynamic equilibrium of the system is not perturbed. Recently, several approaches which go in this direction have been proposed, ${ }^{68,102-104}$ they differ mainly in the way the thermodynamic equilibrium is ensured. Here we describe the AdResS ${ }^{71-74}$ method, whose basic ingredients are: (i) the introduction of a transition region, between the atomistic and coarse grained, characterized by a switching function $w(x)$ that is zero in the coarse-grained region and one in the atomistic region and continuous and monotonic in between (see also Fig. 2) and (ii) the interpolation via $w(x)$ of the atomistic and coarse-grained forces:

$$
\boldsymbol{F}_{\alpha \beta}=w\left(x_{\alpha}\right) w\left(x_{\beta}\right) \boldsymbol{F}_{\alpha \beta}^{\mathrm{atom}}+\left[1-w\left(x_{\alpha}\right) w\left(x_{\beta}\right)\right] \boldsymbol{F}_{\alpha \beta}^{\mathrm{CG}}
$$

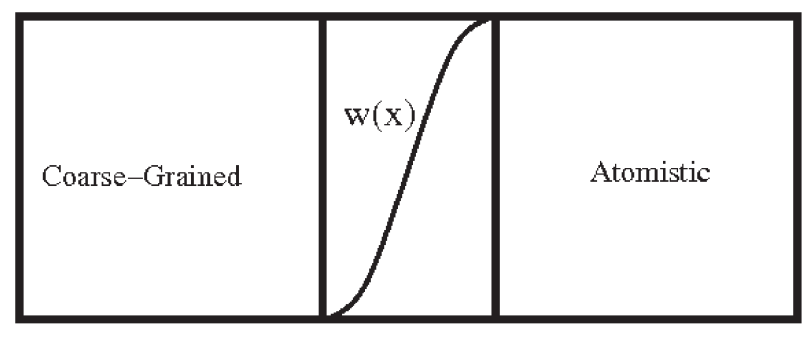

$\triangle$

Fig. 2 Schematic picture of the AdResS box. Atomistic region on the right, coarse-grained left, and transition region $\Delta$ in between. $w(x)$ is the switching function for the transition from a coarse grained to an atomistic resolution and vice versa. An example of a tetrahedral molecule (test system) that changes according to $w(x)$ is shown in Fig. 3. This representation is taken from ref. 71 . rather than the potentials. $\alpha$ and $\beta$ indicate two distinct molecules, $\boldsymbol{F}_{\alpha \beta}^{\text {atom }}$ is obtained from the atomistic potential where atoms of molecule $\alpha$ interact with those of $\beta$, and $\boldsymbol{F}_{\alpha \beta}^{\mathrm{CG}}$ is obtained from the coarse-grained pair potential between the centre of mass of the $\alpha$ and that of $\beta$. In practice, eqn (35) leads to a smooth transition from atomistic to coarse-grained trajectories without a major perturbation of the overall evolution of the system. A very important point of eqn (35) is that, by construction, Newton's Third Law is obeyed. However, this adaptive force alone cannot ensure thermodynamic equilibrium, because it is not conservative; a potential energy cannot be written explicitly, thus one cannot ensure conservation of energy and as a consequence cannot have a clear control on the equilibrium of the system. The problem is basically that one couples different molecular resolutions (atomistic, coarse-grained and a continuous sequence of hybrid resolutions in the transition region) each being characterized by its own (intrinsic) chemical potential. As a consequence, if one starts from a homogeneous density (the stationary state of the full atomistic simulation), the system will evolve towards a stationary state with nonhomogeneous density because the fugacity at the starting stage is not uniform. This of course is not wanted since the AdResS simulation should reproduce the uniform density of the full atomistic system (target system). This problem has been solved by the introduction of a thermodynamic force and the coupling to a local thermostat. The role of the thermostat is that of providing, locally, the amount of kinetic energy required by the slowly introduced degrees of freedom to be in equilibrium with the surrounding as the molecule crosses the transition region. This slow process of acquiring degrees of freedom and being at any instant in equilibrium with the surrounding allows the molecule to enter into the atomistic region without encountering kinetic barriers. The temperature in the transition region is defined by the extension of the equipartition theorem to fractional degrees of freedom. ${ }^{73,74}$ The tools mentioned above are derived from basic principles of thermodynamics and statistical mechanics and have been proven to be rather robust in assuring the overall thermodynamic equilibrium to the AdResS simulations even for the delicate situation of binary mixtures. ${ }^{105}$ To overcome the problem of lack of Hamiltonian, one may think of applying the interpolation formula on Hamiltonians instead of forces, however this has been shown to lead to the violation of physical or mathematical principles. ${ }^{106}$ The force interpolation scheme of AdResS has been applied to several systems such as polymers in solution, ${ }^{107}$ liquid water ${ }^{108,109}$ and extended to the coupling with the continuum. ${ }^{110,111}$ Recently the solvation of a series of bucky balls, $\mathrm{C}_{60}-\mathrm{C}_{2160}$, in water has been studied with AdResS. ${ }^{112}$ Special attention deserves the extension to quantum problems. In general an adaptive quantum-classical approach would require not only the change of the number of degrees of freedom but also of the physical principle involved. In fact while classical mechanics is governed by a deterministic evolution, quantum mechanics is characterized by the probabilistic nature. For cases involving electrons, a proper classical-quantum adaptive scheme based on the Schrödinger equation must be able to deal with the problem of a variable number of particles and thus a varying particle normalization 


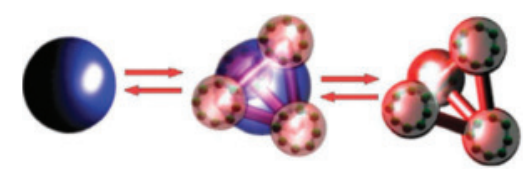

Fig. 3 Adaptive resolution from path integral (ring polymer) representation to coarse-grained through a continuous sequence of intermediate resolution for the tetrahedral molecule. A liquid of such molecules was studied with this adaptive resolution; results show that the equilibrium structures in the quantum (path integral) region are the same as in a full path integral simulation. This representation is taken from ref. 70 .

condition as the system evolves. So far, schemes that treat this case are based on practical solutions without providing a complete and consistent theoretical framework. ${ }^{67,69}$ Instead, if the quantum particles are the atoms without considering the electrons explicitly, then the quantum problem can be mapped on an effective classical one. Thus, as a matter of fact, the adaptive coupling occurs between classical descriptions. The idea is based on the path integral description of atoms in which a quantum atom is represented as a classical polymer ring. In this context, the beads of the polymer ring are fictitious classical particles. This means that in one region one can have an atomistic or coarse-grained resolution and in another a path integral resolution where each atom is represented by a polymer ring. In this situation the principles of AdResS apply straightforwardly as to the case of two classical regions characterized by different numbers of degrees of freedom. The application to study the equilibrium statistical properties of a liquid of tetrahedral molecules has shown that indeed this idea is rather robust both conceptually and numerically ${ }^{70}$ (see also Fig. 3).

\section{Results and discussion}

\section{A. Azobenzene in the gas phase}

1. Force field development. Using the GROMOS 45a 3 force field (see Section II C) as a starting point to derive a new force field suitable for $\mathrm{AB}$, we adjusted only the bonded parameters and the charges of the azo group while keeping the original values for the remaining parameters. The parametrization was carried out in such a way as to achieve maximum agreement between the dynamical distributions obtained from force field ( $\mathrm{MM})$ and $a b$ initio molecular dynamics (QM) simulations in the gas phase at $300 \mathrm{~K}$ concerning the relevant bond lengths, bond angles and dihedral angles. This ensures maximum compatibility between the QM and MM descriptions - so that switching adaptively between the two descriptions for a given $\mathrm{AB}$ unit is as smooth as possible in future adaptive multiscale applications.

Table 1 summarizes our results for the non-standard parameters. In all cases, the values for the force constants are adapted to reproduce the widths of the distributions of bond lengths, angle and dihedrals, starting out from the force field's standard values for chemically similar internal coordinates. In this spirit, we also decided to use the same force constants and point charges for the cis and trans isomers; only the equilibrium reference values for the bonded potentials differ.
Table 1 Non-standard force field parameters derived for azobenzene. For the azobenzene structure and atomic numbering scheme see Fig. 4a (X denotes any atom)

\begin{tabular}{llll}
\hline Entity & & Force constant & Reference \\
\hline Bonds: & $\mathrm{NN}$ & $1.40 \times 10^{3} \mathrm{~kJ}\left(\mathrm{~mol} \mathrm{\AA}^{4}\right)^{-1}$ & $1.2625 \AA$ \\
& $\mathrm{CN}$ & $0.72 \times 10^{3}{\mathrm{~kJ}\left(\mathrm{~mol}^{4}\right)^{-1}}^{-1.4325 \AA}$ \\
Angles: & $\mathrm{CNN}$ & $650.0 \mathrm{~kJ} \mathrm{~mol}^{-1}$ & $116.5^{\circ}$ \\
& $\mathrm{CCN}$ & $560.0 \mathrm{~kJ} \mathrm{~mol}^{-1}$ & $120.0^{\circ}$ \\
Dihedrals: & $\mathrm{CNNC}$ & $70.0 \mathrm{~kJ} \mathrm{~mol}^{-1}$ & $180.0^{\circ}$ \\
& $\mathrm{CCNN}$ & $6.0 \mathrm{~kJ} \mathrm{~mol}^{-1}$ & $180.0^{\circ}$ \\
& $\mathrm{XCCX}$ & $40.0 \mathrm{~kJ} \mathrm{~mol}^{-1}$ & $180.0^{\circ}$ \\
Point charges: & $\mathrm{N}$ & & $-0.20 e$ \\
& $\mathrm{C}^{(1)}$ & & $0.20 e$ \\
& $\mathrm{C}^{(2)}-\mathrm{C}^{(6)}$ & & $-0.10 e$ \\
& $\mathrm{H}$ & & $0.10 e$ \\
\hline & & &
\end{tabular}

The point charges for the atoms $\mathrm{C}^{(1)}$ and $\mathrm{N}$ were adapted from average RESP charges ${ }^{113}$ computed along the QM reference trajectories. Since the values obtained for the aromatic ring atoms $\mathrm{C}^{(2)}-\mathrm{C}^{(6)}$ and the hydrogen atoms were close to their standard force field values, we decided to use the standard values and thus to take advantage of the resulting small charge groups. Note that in contrast to the GROMOS convention but in line with the AMBER convention ${ }^{114}$ and in order to distribute the forces evenly over all contributing atoms we define explicitly all dihedral angles involving the two $\mathrm{CN}$ bonds, i.e. the dihedral angles $\mathrm{C}^{(6)} \mathrm{C}^{(1)} \mathrm{NN}^{\prime}$, $\mathrm{C}^{(2)} \mathrm{C}^{(1)} \mathrm{NN}^{\prime}, \quad \mathrm{C}^{\left(6^{\prime}\right)} \mathrm{C}^{\left(1^{\prime}\right)} \mathrm{N}^{\prime} \mathrm{N}, \quad$ and $\quad \mathrm{C}^{\left(2^{\prime}\right)} \mathrm{C}^{\left(1^{\prime}\right)} \mathrm{N}^{\prime} \mathrm{N}$, which consequently results in smaller force constants per dihedral compared to standard force field values.

It was our aim to derive a single, unified force field for $c i s$ and trans which can be easily applied to study, for instance, a mixture of trans and cis $\mathrm{AB}$ molecules in the condensed phase. In addition, such a force field does not need to be modified during a simulation once a photoinduced cis $\leftrightarrow$ trans isomerisation has occurred in a preceding nonadiabatic QM/MM simulation. Therefore, in the applications described below, we employ the average values determined for cis and trans (see Table 1). This is a minor approximation, as the only differences between the two isomers concern the $\mathrm{NN}$ and $\mathrm{CN}$ bond lengths and the $\mathrm{CNN}$ bond angle, which are merely $0.015 \AA$ and $5^{\circ}$, respectively.

The most difficult parameter to adjust was the force constant for the dihedral angle $\angle C N N C$. Here we settled for the best compromise between sufficient dihedral flexibility and a high enough barrier for the thermal trans $\leftrightarrow$ cis isomerization in the ground state. Our new force field yields a barrier along the torsional reaction coordinate of $\sim 140 \mathrm{~kJ} \mathrm{~mol}^{-1}$, in reasonable agreement with recent $a b$ initio calculations ${ }^{115}$ predicting $\sim 160 \mathrm{~kJ} \mathrm{~mol}^{-1}$. Increasing the barrier in the force field would yield to an even narrower distribution function for the dihedral CNNC angles. On the other hand, the presently parametrized barrier height is sufficiently large to prevent thermally induced cis $\leftrightarrow$ trans isomerizations in the simulations of bulk $\mathrm{AB}$ at $400 \mathrm{~K}$ (corresponding to an energy of about $3.3 \mathrm{~kJ} \mathrm{~mol}^{-1}$ ) (see Section III B 1), which will not occur on the timescale accessible to classical simulation.

The new force field also reproduces the energy difference between the cis and trans isomers reasonably well. It predicts 

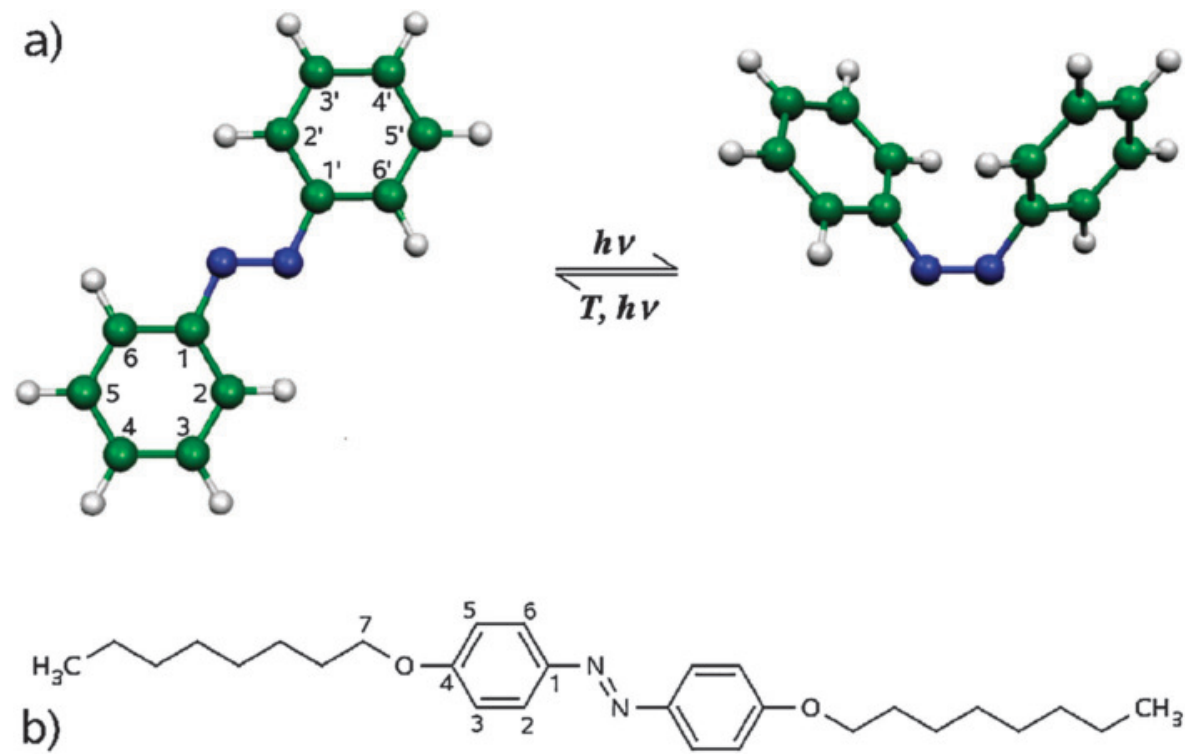

Fig. 4 (a) Structure and atom numbering scheme of trans- and cis-azobenzene in the left and right panels, respectively. (b) Chemical structure of $4,4^{\prime}$-dioctyloxy-azobenzene (8AB8).

Table 2 Extension of azobenzene force field to include ether linkage $\mathrm{C}^{(4)}-\mathrm{O}-\mathrm{C}^{(7)}$ (see Fig. 4 for structure and atomic numbering scheme)

\begin{tabular}{llll}
\hline Entity & & Force constant & Reference value \\
\hline Bonds: & $\mathrm{OC}^{(7)}$ & $8.18 \times 10^{2}{\mathrm{~kJ}\left(\mathrm{~mol}^{4}\right)^{-1}}^{-1}$ & $1.430 \AA$ \\
& $\mathrm{C}^{(4)} \mathrm{O}$ & $1.02 \times 10^{3} \mathrm{~kJ}^{4} \mathrm{~mol} \mathrm{~A}^{-1}$ & $1.360 \AA$ \\
Angle: & $\mathrm{C}^{(4)} \mathrm{OC}^{(7)}$ & $620.0 \mathrm{~kJ} \mathrm{~mol}^{-1}$ & $116.0^{\circ}$ \\
Dihedral: & $\mathrm{C}^{(3,5)} \mathrm{C}^{(4)} \mathrm{OC}^{(7)}$ & $6.0 \mathrm{~kJ} \mathrm{~mol}^{-1}$ & $180.0^{\circ}$ \\
Point charges: & $\mathrm{O}$ & & $-0.332 e$ \\
& $\mathrm{C}^{(7)}$ & & $0.178 e$ \\
\hline
\end{tabular}

the cis structure to be higher in energy by $36 \mathrm{~kJ} \mathrm{~mol}^{-1}$, which is close to the value of $50 \mathrm{~kJ} \mathrm{~mol}^{-1}$ obtained experimentally ${ }^{116}$ and from CASPT2 ab initio calculations. ${ }^{115}$

A detailed comparison of dynamical and optimised structural data from QM and MM calculations can be found in ref. 46. The data underline the good quality of our parameter set for trans- and cis-AB, respectively.

Based on this force field for the $\mathrm{AB}$ chromophore we have extended the set of force field parameters to be able to study materials containing this photoswitch such as $8 \mathrm{AB} 8$, introduced in Fig. 4, where aliphatic side chains are attached to the phenyl rings of $\mathrm{AB}$ via ether bridges. We used methylphenyl-ether $\left(\mathrm{H}_{3} \mathrm{C}-\mathrm{O}-\mathrm{C}_{6} \mathrm{H}_{5}\right)$ as a model system to derive the force field parameters necessary to describe the $\mathrm{C}^{(4)}-\mathrm{O}-\mathrm{C}^{(7)}$ link unit (see Fig. 4 for the atomic numbering scheme) whereas the remainder of these side chains are treated using standard force field parameters.

As for AB itself, a Car-Parrinello run at $300 \mathrm{~K}$ was performed with $\mathrm{H}_{3} \mathrm{C}-\mathrm{O}-\mathrm{C}_{6} \mathrm{H}_{5}$ as the QM reference with the aim to parametrize those internal coordinates that involve the oxygen atom of the ether group. In order to take into account dynamical fluctuations of the $\mathrm{C}^{(5)} \mathrm{C}^{(4)} \mathrm{OC}(7)$ dihedral angle, RESP charges for $\mathrm{C}^{(4)}$, $\mathrm{O}$, and $\mathrm{C}^{(7)}$ were calculated for different angles between $0^{\circ}$ and $90^{\circ}$ in steps of $10^{\circ}$. The resulting charge of the methyl group is taken as the charge of the alkyl carbon atom $\mathrm{C}^{(7)}$ (united atom approach), and the resulting charge of the aryl carbon atom $\mathrm{C}^{(4)}$ is adjusted so as to yield a neutral $\mathrm{C}^{(4)}-\mathrm{O}-\mathrm{C}^{(7)}$ unit. Force field point charges were then obtained by Boltzmann averaging over the torsionangle dependent RESP charges. The resulting parameters for the ether linkage are collected in Table 2.

2. Photoisomerisation of azobenzene. The photoisomerisation of $\mathrm{AB}$ was studied using the nonadiabatic AIMD method introduced in Section II A 3. Ten surface hopping trajectories were calculated for both directions, trans $\rightarrow$ cis and cis $\rightarrow$ trans, sampling the initial conditions randomly from ground state AIMD runs at $300 \mathrm{~K}$.

a. Potential energy landscapes. It is important for the discussion below of the photoisomerisation dynamics and mechanism to determine first the shape of the ground and excited state potential landscapes, and to assess the quality of the ROKS $S_{1}$ PES used in the na-AIMD and na-QM/MM simulations. We have therefore calculated ROKS, CASSCF, and CASPT2 energy profiles along the CNNC dihedral angle, which is an important internal coordinate for photoisomerisation in the $S_{1}$ state. ${ }^{63}$ On the left hand side of Fig. 5 we present DFT, CASSCF, and CASPT2 $S_{0}$ and ROKS, CASSCF, and CASPT2 $S_{1}$ potential energy curves for the DFT ground state minimum energy path (MEP) along the CNNC dihedral angle, $\theta$. Note that the ROKS energies have been corrected upwards 

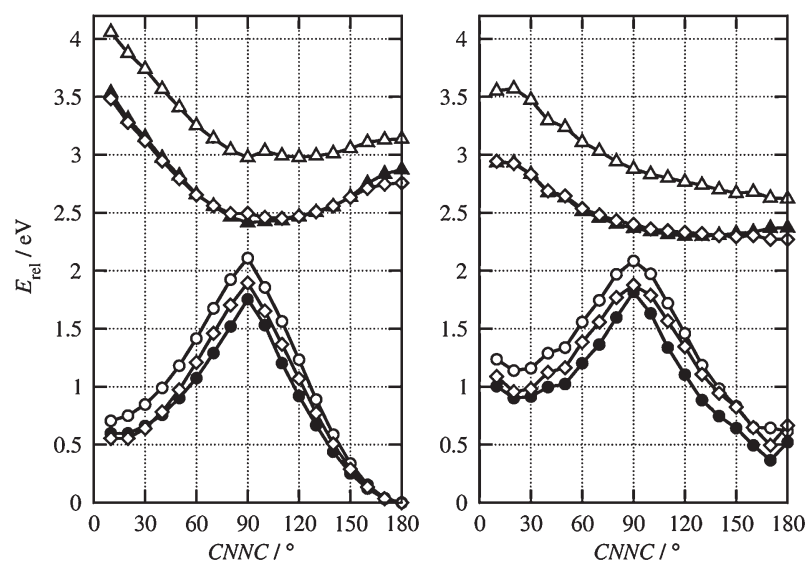

Fig. $5 S_{0}$ and $S_{1}$ energy profiles along the CNNC dihedral angle using structures optimized using DFT (PBE) for the $S_{0}$ ground state (left) and ROKS (PBE) for the $S_{1}$ first excited state (right). The ROKS energies $(\boldsymbol{\Lambda}-\mathbf{\Delta})$ are compared to the state-averaged CAS $(14,12)$ $(\Delta-\Delta)$ and CASPT2 $(\diamond \longrightarrow)$ data. $S_{0}$ ground state energies are shown for DFT $(-\bullet)$, state-averaged $\operatorname{CAS}(14,12)\left(\mathrm{O}_{-} \mathrm{O}\right)$ and CASPT2 $(\diamond \longrightarrow)$. All energies are relative to the respective $S_{0}$ energies of trans$\mathrm{AB}$ optimized with DFT in the $S_{0}$ state; $0.7 \mathrm{eV}$ have been added to the ROKS energies as explained in Section III A 2a.

by a constant shift of $0.7 \mathrm{eV}$ which was originally determined from the difference between ROKS and experimental vertical excitation energies. ${ }^{63}$ All ground state curves in Fig. 5 have a maximum at $90^{\circ}$, the DFT curve being very close to the CASPT2 curve, while CASSCF is seen to overestimate by about $0.2 \mathrm{eV}$ compared to CASPT2 over a wide range of $\theta$. The agreement between the ROKS and CASPT2 excited state curves throughout the entire range of the isomerization coordinate $\theta$ is remarkable, whereas the CASSCF $S_{1}$ curve is very similar in shape but shifted upwards by about $0.5 \mathrm{eV}$.

The right hand side of Fig. 5 shows ground and excited state energy profiles along the ROKS optimised $S_{1}$ excited state MEP along the CNNC dihedral. It is seen that the ROKS curve has a shallow $S_{1}$ minimum around $\theta \approx 120^{\circ}$ which can be reached by a barrierless path from both the cis and trans Franck-Condon (FC) points at $\theta=10^{\circ}$ and $\theta=180^{\circ}$, respectively. As for the $S_{0} \mathrm{MEP}$, there is again striking agreement between the ROKS and CASPT2 excited state curves, while the CASSCF energies are higher than the CASPT2 data by $0.3-0.5 \mathrm{eV}$. With regards to the differences between the trans $\rightarrow$ cis and cis $\rightarrow$ trans photoisomerisation dynamics which we shall discuss below, it is important to realise that there is a considerably larger potential energy difference between the FC point and the $S_{1}$ global minimum upon vertical excitation of the cis isomer as compared to trans-AB.

At this stage it is concluded, based on the direct comparison to CASPT2 reference data in Fig. 5, that the aforementioned constant blue-shift applied to the ROKS data indeed corrects consistently the gap not only at $90^{\circ}$ but along the entire MEPs along the $\mathrm{CNNC}$ dihedral angle, which is a major component of the reaction coordinate of $\mathrm{AB}$ photoisomerization, both in the $S_{0}$ and in the $S_{1}$ in the full range between $0^{\circ}$ and $180^{\circ}$.

b. Definition of internal coordinates. To analyze in detail the isomerisation mechanism and possible differences between the gas and the liquid phase, we describe internal motion in terms of the plane normal vectors $\boldsymbol{n}^{\mathrm{R}}$ and $\boldsymbol{n}^{\mathrm{R}^{\prime}}$ of the two aromatic rings at their geometric centres, $\mathrm{R}$ and $\mathrm{R}^{\prime}$, together with the normal vectors $\boldsymbol{n}^{\mathrm{N}}$ and $\boldsymbol{n}^{\mathrm{N}^{\prime}}$ of the $\mathrm{C}^{(1)} \mathrm{NN}^{\prime}$ and $\mathrm{NN}^{\prime} \mathrm{C}^{\left(1^{\prime}\right)}$ coordination planes at $\mathrm{N}$ and $\mathrm{N}^{\prime}$. To measure torsion of the $\mathrm{C}^{(1)} \mathrm{NN}^{\prime}$ and $\mathrm{NN}^{\prime} \mathrm{C}^{\left(1^{\prime}\right)}$ coordination planes, we define an intrinsic (right-handed) coordinate system whose origin is at the geometric midpoint of the two nitrogen atoms, the $x$-axis is parallel to the $\mathrm{N}=\mathrm{N}^{\prime}$ bond and the $z$-axis is parallel to the arithmetic mean of $n^{\mathrm{N}}$ and $n^{\mathrm{N}^{\prime}}$. The absolute torsion is then monitored as the change in angle of the projection of the normal vector $n^{\alpha}$ onto the $y z$ plane, $n_{y z}^{\alpha}, \psi^{\alpha}(t)=\angle\left(\boldsymbol{n}_{y z}^{\alpha}(\mathrm{t}), n_{y z}^{\alpha}(0)\right)$, while $\psi^{\alpha \beta}(t)=\angle\left(\boldsymbol{n}^{\alpha}(t), \boldsymbol{n}^{\beta}(t)\right)$, with $\alpha, \beta \in\left\{\mathbf{N}, \mathbf{N}^{\prime}, \mathbf{R}, \mathbf{R}^{\prime}\right\}$, gives relative changes, e.g. $\psi^{\mathrm{NN}^{\prime}}$ is the $\mathrm{C}^{(1)} \mathrm{NN}^{\prime} \mathrm{C}^{\left(1^{\prime}\right)}$ dihedral angle and $\psi^{\mathrm{RN}}$ captures rotation of the phenyl rings.

c. cis $\rightarrow$ trans. In this section, we analyze in detail the mechanism of cis-AB to trans-AB photoisomerisation. In Fig. 6a we present the time evolution of $\psi^{\mathrm{NN}^{\prime}}$ (i.e. the CNNC dihedral) for a typical cis $\rightarrow$ trans trajectory together with the corresponding time evolution of $\psi^{\mathrm{N}}$ and $\psi^{\mathrm{N}^{\prime}}$. It is seen that $\psi^{\mathrm{NN}^{\prime}}$ initially changes rapidly and after about $30 \mathrm{fs}$ reaches a value of $\sim 90^{\circ}$. After the $S_{1} \rightarrow S_{0}$ transition to the ground state, $\psi^{\mathrm{NN}^{\prime}}$ reaches a value of $\sim 180^{\circ}$, thus indicating a successful cis $\rightarrow$ trans isomerisation. Inspection of the order parameters $\psi^{\mathrm{N}}$ and $\psi^{\mathrm{N}^{\prime}}$ (cf. Fig. 6a) reveals that the total change in $\psi^{\mathrm{NN}^{\prime}}$ is due to equal contributions from the two coordination planes at $\mathrm{N}$ and $\mathrm{N}^{\prime}$ in opposite directions. Photoisomerisation is dominated by a pedal motion of the CNNC group and not by large amplitude rotation of the phenyl rings. This is illustrated by the inset of Fig. 6a which shows the rmsd's of the $\mathrm{N}$ atoms and the phenyl ring centres $\mathrm{R}$. We can see that during the first $30 \mathrm{fs}$ it is mainly the translocation of the $\mathrm{N}$ atoms that is responsible for the change in $\psi^{\mathrm{NN}^{\prime}}$ by $\sim 90^{\circ}$, while the phenyl rings remain largely fixed in space. Fig. 7 shows the ensemble averaged changes of the $\mathrm{CNNC}$ angle (i.e. $\psi^{\mathrm{NN}^{\prime}}$ ) as a function of time after vertical

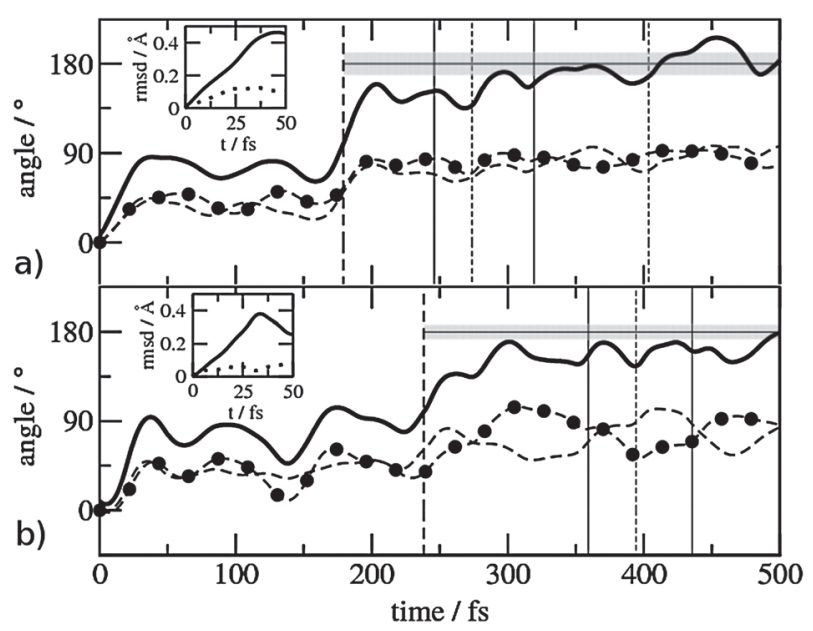

Fig. 6 Time evolution of $\psi^{\mathrm{NN}^{\prime}}(-), \psi^{\mathrm{N}}(--)$, and $-\psi^{\mathrm{N}^{\prime}}(-\mathbf{O})$ for typical cis $\rightarrow$ trans trajectories in the gas phase (a) and the liquid (b). Vertical lines indicate $S_{1} \rightarrow S_{0}(--)$ and $S_{0} \rightarrow S_{1}(-)$ hops. Grey bars indicate the $\psi^{\mathrm{NN}^{\prime}}$ trans reference range. The insets show the rmsd's of $\mathrm{N}, \mathrm{N}^{\prime}$ (solid) and $\mathrm{R}, \mathrm{R}^{\prime}$ (dashed) relative to $t=0$. 


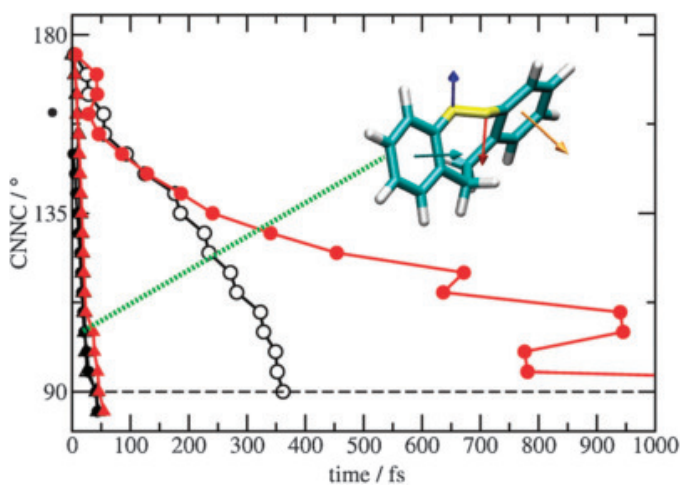

Fig. 7 Ensemble average of the CNNC dihedral angle, $\psi^{\mathrm{NN}^{\prime}}(t)$, during time-evolution in the first excited state, $S_{1}$, where the specific value $\psi^{\mathrm{NN}^{\prime}}$ at time $t$ is reached for the first time after vertical photoexcitation from $S_{0}$ at $t=0$ : gas phase trans-AB- $\mathrm{C}_{2}(-\bullet)$, gas phase trans-AB $(\mathrm{O}-\mathrm{O})$, gas phase $c i s-\mathrm{AB}(\Delta-\Delta)$, liquid phase trans-AB $(\bullet-\bullet)$, liquid phase cis-AB $(\boldsymbol{\Delta}-\mathbf{\Delta})$. In the case of cis-AB, $180-\psi^{\mathrm{NN}}$ is plotted. The perpendicular conformation, i.e. $\psi^{\mathrm{NN}^{\prime}}=90^{\circ}$, is marked by the horizontal dashed line. For liquid trans-AB, the non-monotonicity arises from the fact that ensemble averages were obtained with different numbers of trajectories. The inset shows the optimised structure of trans-AB- $\mathrm{C}_{2}$; normal vectors indicate the orientation of the phenyl rings, $n^{\mathrm{R}}$ and $n^{\mathrm{R}^{\prime}}$ (cyan, orange), and of the CNN coordination plane of the N atoms, $\boldsymbol{n}^{\mathrm{N}}$ and $\boldsymbol{n}^{\mathrm{N}^{\prime}}$ (red, blue).

photoexcitation. The average time it takes for a molecule to reach $\psi^{\mathrm{NN}^{\prime}}=90^{\circ}$ is just $42 \mathrm{fs}$.

d. trans $\rightarrow$ cis. The time evolution of $\psi^{\mathrm{NN}^{\prime}}$ in the trans $\rightarrow$ cis case is displayed in Fig. 8a. It shows a more or less smooth decrease from $180^{\circ}$ at $t=0$ to $\sim 90^{\circ}$ at $t \approx 300 \mathrm{fs}$, where a $S_{1} \rightarrow S_{0}$ transition occurs. After the hop $\psi^{\mathrm{NN}^{\prime}}$ rapidly falls to a value close to $0^{\circ}$, thus indicating a successful trans $\rightarrow$ cis isomerisation. As observed for the cis $\rightarrow$ trans isomerisation, the total change of $\sim 180^{\circ}$ in $\psi^{\mathrm{NN}^{\prime}}$ is eventually accomplished by equal contributions $\left(\sim 90^{\circ}\right)$ from $\psi^{\mathrm{N}}$ and $\psi^{\mathrm{N}^{\prime}}$ (dashed and circled lines), in opposite directions. Interestingly, however,

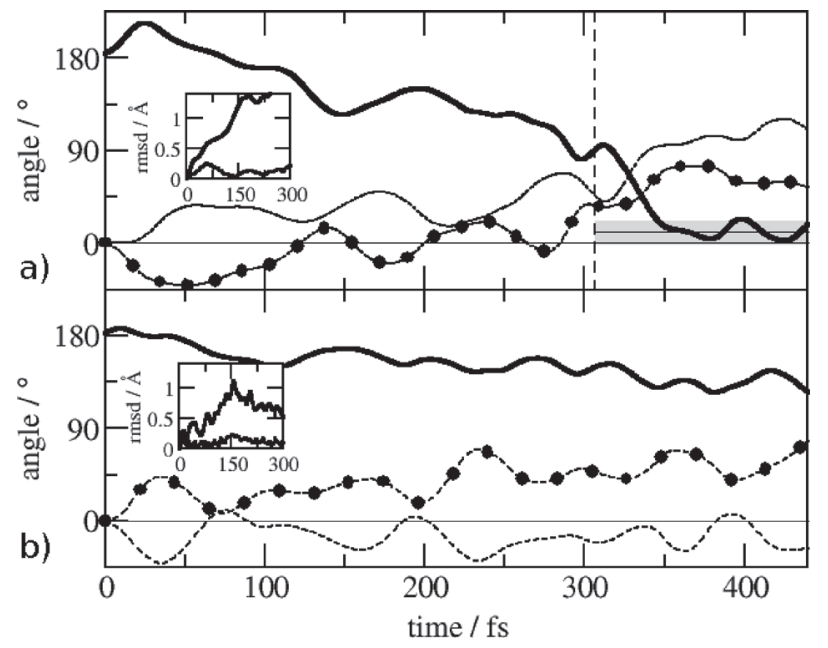

Fig. 8 Time evolution of $\psi^{\mathrm{NN}^{\prime}}(-), \psi^{\mathrm{N}}(--)$, and $-\psi^{\mathrm{N}^{\prime}}(-0)$ for typical trans $\rightarrow$ cis trajectories in the gas phase (a) and in the liquid (b). Grey bars indicate the $\psi^{\mathrm{NN}^{\prime}}$ cis reference range. See the caption of Fig. 6. initially both nitrogen coordination planes rotate in the same direction, thus producing no net change of $\psi^{\mathrm{NN}^{\prime}}$ (e.g. at $t=50 \mathrm{fs}$ ). This is a crucial difference from the cis $\rightarrow$ trans photoisomerisation mechanism.

As for the cis $\rightarrow$ trans isomerisation, the change in $\psi^{\mathrm{NN}^{\prime}}$ is produced by the translocation of the $\mathrm{N}$ atoms, again indicating a pedal motion-like mechanism (see the inset of Fig. 8a). Interestingly, a similar translocation at fixed $\psi^{\mathrm{NN}^{\prime}}$ has been found in X-ray diffraction experiments of azobenzene crystals. $^{117}$

Being the characteristic feature also of the trans $\rightarrow$ cis photoisomerisation, the pedal motion of the two $\mathrm{N}$ atoms accounts for the experimental finding of fast isomerisation dynamics in rotation-restricted trans- $\mathrm{AB}^{118}$ and clearly rules out an inversion-type mechanism as deduced from resonance Raman intensity analysis. ${ }^{119}$ Note that there is no largeamplitude rotation of the phenyl rings involved as has been suggested for the interpretation of fluorescence anisotropy data. $^{120}$

The trans $\rightarrow$ cis photoisomerisation is significantly slower than $c i s \rightarrow$ trans. This is illustrated by the ensemble averaged changes of the CNNC angle shown in Fig. 7. The average time it takes for a molecule to reach $\psi^{\mathrm{NN}^{\prime}}=90^{\circ}$ is about $360 \mathrm{fs}$, one order of magnitude longer than that for cis $\rightarrow$ trans. This observation is in accord with the longer $S_{1}$ lifetimes measured experimentally for trans-AB compared to cis-AB. ${ }^{121}$

3. Photoisomerisation of chemically bridged azobenzene. Recently, a greatly enhanced trans $\rightarrow$ cis quantum yield $\Phi_{\text {trans } \rightarrow \text { cis }}^{\mathrm{AB}-\mathrm{C}_{2}}$ was reported ${ }^{122}$ for a bridged azobenzene $\left(\mathrm{AB}-\mathrm{C}_{2}\right.$ in Fig. 7) in the $S_{1}$ state as compared to the parent molecule $\mathrm{AB}$. This finding seemed surprising at first as the structural changes involved in isomerisation should be expected to be hindered by the restriction due to the presence of a bridge interconnecting the phenyl rings. Subsequent na-AIMD simulations ${ }^{123}$ then demonstrated that counterintuitively the bridge does not hinder photoisomerization. On the contrary, it suitably pre-orients the phenyl rings such that $A B-C_{2}$ can more easily undergo trans $\rightarrow$ cis isomerization thus yielding not only an enhanced quantum yield $\Phi_{\text {trans } \rightarrow \text { cis }}^{\mathrm{AB}-\mathrm{C}_{2}}$ but also ultra-short $S_{1}$ lifetimes.

Upon chemical modification of $\mathrm{AB}$ to form $\mathrm{AB}-\mathrm{C}_{2}$ (i.e. addition of a $-\mathrm{CH}_{2}-\mathrm{CH}_{2}-$ bridge in the ortho position of the phenyl rings) the trans isomer becomes nonplanar, due to the orientation of the phenyl rings, while only minor structural changes arise for the cis isomer (Fig. 7). The mechanical strain introduced into trans-AB- $\mathrm{C}_{2}$ makes it less stable than cis- $\mathrm{AB}-\mathrm{C}_{2}$ by $0.31 \mathrm{eV}$ according to DFT (which again compares favourably to the CASPT2 value of $0.35 \mathrm{eV}$ ).

Performing nonadiabatic AIMD simulations after vertical $S_{0} \rightarrow S_{1}$ photoexcitation of trans-AB- $C_{2}$ roughly half of them are found to result in successful trans $\rightarrow$ cis isomerization, the quantum yield being, more precisely, $\Phi_{\text {trans } \rightarrow \text { cis }}^{\mathrm{AB}-\mathrm{C}_{2}}=(47 \pm 10) \%$ including the statistical error of the sample computed using the blocking method. ${ }^{124}$ The computed number is consistent with the experimental finding ${ }^{122}$ of $\Phi_{\text {trans } \rightarrow \text { cis }}^{\mathrm{AB}_{2}-\mathrm{C}_{2}}=(50 \pm 10) \%$. It is noted in passing that the surface hopping method applied here is known to overemphasize coherence, ${ }^{82,125}$ which is a potential source of error when extracting quantitative 
information such as quantum yields or excited state lifetimes. However, it has been shown ${ }^{126}$ to perform rather well when compared to other approximate methods suitable to simulate complex molecular systems and, moreover, it has been demonstrated ${ }^{127}$ that surface hopping quantum yields are only slightly underestimated for bare $\mathrm{AB}$ thus validating this approach for the specific case. In stark contrast to trans-AB- $\mathrm{C}_{2}$, the parent compound $\mathrm{AB}$ features a much lower experimental value of $\Phi_{\text {trans } \rightarrow \text { cis }}^{\mathrm{AB}}=24 \%,{ }^{122}$ which is again in harmony with nonadiabatic AIMD where only roughly $20 \%$ of the trajectories yielded isomerization. ${ }^{63}$ Thus, the computational approach reproduces the observed ${ }^{122}$ striking difference between $\mathrm{AB}-\mathrm{C}_{2}$ versus $\mathrm{AB}$ in terms of quantum yields. The calculations demonstrate that photoisomerisation of trans- $\mathrm{AB}-\mathrm{C}_{2}$ is astonishingly fast according to Fig. 7, which shows that trans-AB- $\mathrm{C}_{2}$ reaches the decisive perpendicular conformation, i.e. $\psi^{\mathrm{NN}^{\prime}}=90^{\circ}$, in only $\sim 40 \mathrm{fs}$ which is one order of magnitude faster than that for the corresponding parent trans- $\mathrm{AB}$ and, in fact, comparable to plain cis- $\mathrm{AB}$. Moreover, in contrast to trans- $\mathrm{AB}$, trans- $\mathrm{AB}-\mathrm{C}_{2}$ is found to rapidly reach the region beyond the ground state barrier to trans $\rightarrow$ cis isomerisation along the CNNC coordinate characterized by $\psi^{\mathrm{NN}^{\prime}} \ll 90^{\circ}$, thus entering the cis-AB-C product potential well leading to successful photoisomerization (the optimized value for the cis-AB-C $\mathrm{C}_{2}$ product being $\psi_{0}{ }^{\mathrm{NN}^{\prime}}=$ $6.6^{\circ}$ in $S_{0}$ ). This behavior rationalizes the greatly enhanced isomerization quantum yield found for trans-AB- $\mathrm{C}_{2}$ compared to trans- $\mathrm{AB}$ consistently both in experiment ${ }^{122}$ and in these simulations.

But why is photoisomerization promoted upon bridging? First principles simulations of the photoiomerization of $\mathrm{AB}$ in the bulk ${ }^{62,63}$ (see Section III B 2) and suspended between gold electrodes ${ }^{128,129}$ reveal that spatial confinement and mechanical constraints, respectively, only mildly affect cis $\rightarrow$ trans isomerisation while a pronounced slowing down is seen for trans $\rightarrow$ cis. The reason can be traced back to the ultrafast pedal motion of the $\mathrm{N}$ atoms yielding a CNNC angle of $\psi^{\mathrm{NN}^{\prime}} \approx 90^{\circ}$ in the $S_{1}$, which competes with the need to achieve co-planarity of the $\mathrm{CNN}$ planes with their adjacent rings, $\psi^{\mathrm{RN}} \approx 0^{\circ}$. In this sense, trans- $\mathrm{AB}-\mathrm{C}_{2}$ is very similar to cis $\rightarrow$ trans, since both have a very similar non-co-planar phenyl ring orientation in the $S_{0}$ equilibrium structure $\left(\psi^{\mathrm{RN}}=\psi^{\mathrm{R}^{\prime} \mathrm{N}^{\prime}} \approx 58^{\circ}\right.$ for cis- $\mathrm{AB}$ and $\approx 53^{\circ}$ for trans-AB- $\mathrm{C}_{2}$, see Fig. 7). The energetic reward associated with achieving co-planarity turns out to be a driving force for ultrafast photoisomerisation. The same reasoning can be applied to explain the large difference between trans-AB- $\mathrm{C}_{2}$ and its unbridged parent trans-AB. While trans-AB- $\mathrm{C}_{2}$ tries to compensate the initial non-co-planarity of the phenyl rings and the $\mathrm{CNN}$ planes, trans- $\mathrm{AB}$ is initially planar and therefore completely lacks this incentive.

Hence, the 'bridging' of $\mathrm{AB}$ - commonly viewed as a severe steric hindrance to photoisomerisation-counterintuitively yields a drastically improved photoswitch which isomerizes on a much shorter timescale with a significantly enhanced quantum yield. Extending the same reasoning it is expected that bridging $c i s-\mathrm{AB}$ to yield $c i s-\mathrm{AB}-\mathrm{C}_{2}$ will have only a minor effect on the mechanism and, therefore, the cis $\rightarrow$ trans photoisomerization of $\mathrm{AB}-\mathrm{C}_{2}$ should be as fast as for unbridged $\mathrm{AB}$. This implies that both cis $\rightarrow$ trans and trans $\rightarrow$ cis photoswitching of $\mathrm{AB}-\mathrm{C}_{2}$ should be similarly effective. In fact, experiment predicts a cis $\rightarrow$ trans quantum yield of $72 \pm 4 \%$, even larger than that for trans $\rightarrow$ cis. ${ }^{122} \mathrm{~A}$ recent semiempirical nonadiabatic dynamics study of $\mathrm{AB}-\mathrm{C}_{2}$ has confirmed that nonadiabatic relaxation is ultrafast ( $<100 \mathrm{fs}$ ) for both bridged isomers. ${ }^{130}$ According to those simulations, however, the cis $\rightarrow$ trans quantum yield is only $23 \%$. The authors attribute this underestimation to early surface hops caused by subtle errors in the underlying semiempirical potential energy surfaces.

\section{B. Liquid azobenzene}

1. Classical molecular dynamics in the ground state. Parametrizations of the azo group had been carried out at $300 \mathrm{~K}$ having in mind future applications of the force field at ambient conditions. The present applications to the study of liquid $\mathrm{AB}$ (with a melting point of $341 \mathrm{~K}$ ) and liquid crystalline $\mathrm{AB}$-containing compounds (with phase transition temperatures of $8 \mathrm{AB} 8$ between 372 and $385 \mathrm{~K}$ ) required testing the validity of the classical force field at an increased temperature of $400 \mathrm{~K}$.

We therefore applied the new force field to study liquid $\mathrm{AB}$ at $400 \mathrm{~K}$ and analyze separately the influence of the liquid environment on the structural properties of the cis and trans conformers of $\mathrm{AB}$ by comparison with gas phase simulations at $400 \mathrm{~K}$. As a measure for the extension of the $A B$ unit serves the distribution of the distance between the geometric centres of the two phenyl rings as shown in Fig. 9a and b. For trans- $\mathrm{AB}$ the distributions of the single molecule and the liquid phase are indistinguishable (solid line in Fig. 9a), whereas the conformations of the cis isomer are slightly more affected by the bulk environment (see Fig. 9b). In the liquid phase the cis-AB unit is slightly stretched out compared to the vacuum simulations. Similar observations can be made when analyzing the out-of-plane motions of the phenyl rings by monitoring the distribution functions of the dihedral angle between the normal vectors of the two phenyl rings (data not shown) and of the CCNN dihedral angle (see Fig. 9c and d). The conformations in trans-AB are not affected by the liquid environment (solid line in Fig. 9c), whereas in the case of the cis isomer the amplitude of the ring motion (compared to a planar structure) is slightly larger in the isolated molecule than in the bulk liquid at the same temperature (see Fig. 9d). Additionally, it was found that the distribution functions of the CNNC dihedral angle are not affected by the liquid environment - neither in the case of the trans nor in the case of the cis isomer (data not shown), and it was verified that the system does not undergo thermal cis $\leftrightarrow$ trans isomerisation, which is a rare event that indeed should not occur on the timescale presently accessible by such classical molecular dynamics simulations.

Fig. 9d shows that the distribution of the CCNN dihedral angle in cis-AB has four chemically equivalent maxima around $\pm 54^{\circ}$ and $\pm 126^{\circ}$ and consequently two types of transitions between these states. As indicated in the figure, there is one "fast" type of transition where the phenyl ring is intermediately standing perpendicular to the plane spanned by the $\mathrm{C}^{(1)}$ (or the $\mathrm{C}^{\left(1^{\prime}\right)}$ ) carbon and the two $\mathrm{N}$ atoms, and one 

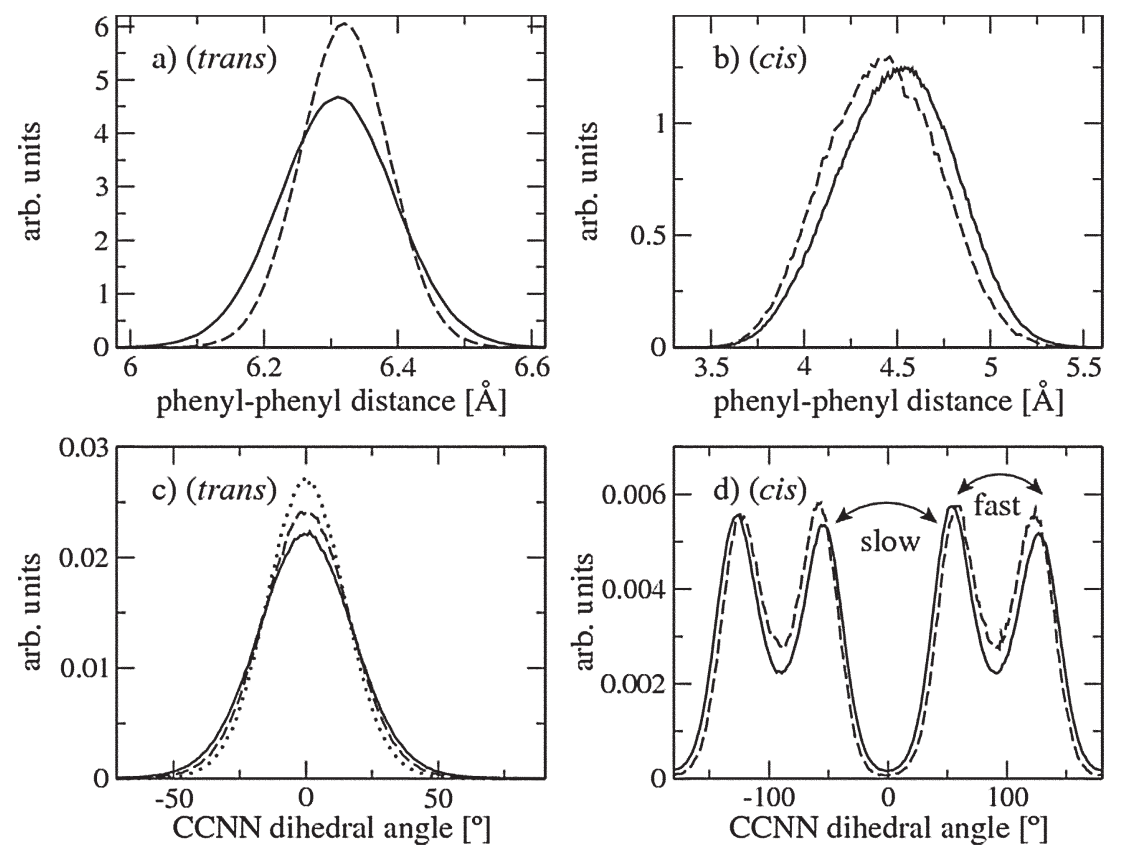

Fig. 9 Distributions of structural parameters of the AB unit under various conditions at $400 \mathrm{~K}$. Panels a and b: distance of the geometrical centres of the two phenyl rings. Panels $\mathrm{c}$ and $\mathrm{d}$ : CCNN dihedral angle. Panel a: trans-AB in the liquid phase as well as in vacuum-solid line, trans-8AB8 in the liquid phase (both isotropic and smectic) and in vacuum-dashed line; panel b: cis-AB in the liquid phase-solid line, cis-AB in vacuum-dashed line; panel c: trans-AB in the liquid phase (equivalent to trans-AB in vacuum and trans $8 \mathrm{AB} 8$ in vacuum) - solid line, trans-8AB8 in isotropic liquid — dashed line, trans-8AB8 in the anisotropic (smectic) phase- dotted line; panel d: cis-AB in the liquid phase- - solid line, cis-AB in vacuum - dashed line.

"slow" type of transition, where the ring is intermediately in-plane with the $\mathrm{C}^{(1)}$ and the two $\mathrm{N}$ atoms (to avoid steric hindrance in this planar conformation during the "slow" transition, the second phenyl ring has to "make way" by adopting a conformation perpendicular to the plane). These transitions are observed in the classical simulations of $c i s-\mathrm{AB}$, whereas the timescale of QM simulations of a few ps is too short to sample these transitions systematically. Fig. 10 shows one example of such a process by monitoring the dynamics of the CCNN dihedral angles of one AB unit in a simulation of liquid cis- $\mathrm{AB}$ at $400 \mathrm{~K}$, where both types of transitions are observed. In addition, snapshots of representative cis-AB

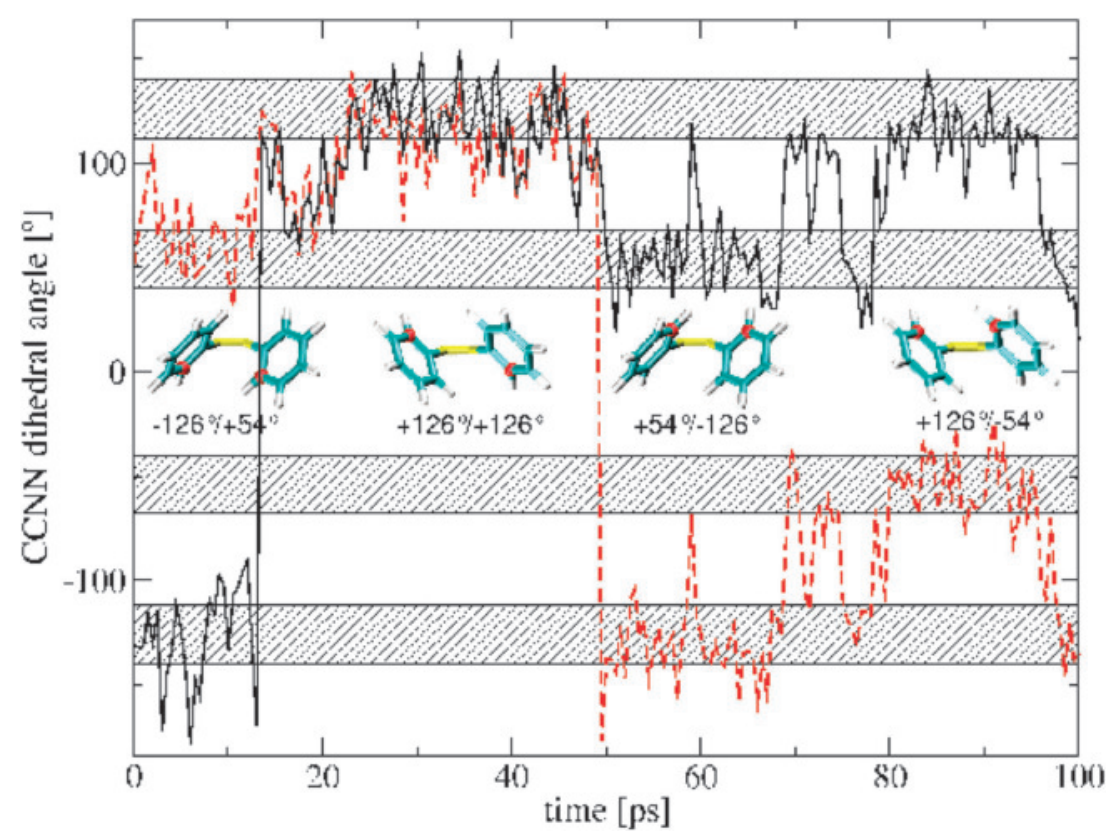

Fig. 10 Time evolution of the CCNN dihedral angles of a cis-AB molecule in liquid environment at $400 \mathrm{~K}$. The striped bars indicate the regions ( $\pm 14^{\circ}$ around the maxima of the distributions at $\pm 54^{\circ}$ and $\pm 126^{\circ}$, see Fig. 9d) used to count the transitions between the states (see text). Snapshots of typical conformations are included with the $\mathrm{C}^{(2)}$ and $\mathrm{C}^{\left(2^{\prime}\right)}$ carbon atoms that are used to define the CCNN dihedral angles marked in red. 
conformations are shown to illustrate the conformational changes during the transitions. In order to get a rough estimate for the timescale of these ring flips the transitions of both types are counted for all CCNN dihedrals in a simulation of 343 cis-AB molecules at $400 \mathrm{~K}$. Since the separation between the states, in particular between the states involved in the "fast" transitions, is ambiguous, narrow regions $\left( \pm 14^{\circ}\right)$ around the maxima of the distributions at $\pm 54^{\circ}$ and $\pm 126^{\circ}$ were defined (as marked in Fig. 10) and only transitions between these regions were counted. This results in transition times of approximately 20 ps for the "fast" and 200 ps for the "slow" ring flips. By Boltzmann inverting the dihedral distribution in Fig. 9d, one obtains an effective barrier for the "fast" transition of the order of about $3 \mathrm{~kJ} \mathrm{~mol}^{-1}$ ( $\sim 1 k_{\mathrm{B}} T$, where $k_{\mathrm{B}}$ is the Boltzmann constant) and for the "slow" transition a barrier of about $12 \mathrm{~kJ} \mathrm{~mol}^{-1}\left(\sim 4 k_{\mathrm{B}} T\right)$, which approximately reproduces the relative magnitude of the two transition rates extracted from the dynamics.

\section{2. $\mathbf{Q M} / \mathbf{M M}$ photoisomerisation simulations}

a. cis $\rightarrow$ trans. The photoisomerisation of $\mathrm{AB}$ in the bulk liquid has been studied using the nonadiabatic QM/MM simulation method introduced in Section II B. Condensed phase effects are investigated by comparing results for the liquid with those for the gas phase (Section III A). Fig. 6b shows the time evolution of $\psi^{\mathrm{NN}^{\prime}}$ (i.e. the CNNC dihedral) for typical cis $\rightarrow$ trans trajectories in the liquid together with the corresponding time evolution of $\psi^{\mathrm{N}}$ and $\psi^{\mathrm{N}^{\prime}}$. Similar to the gas phase (Fig. 6a) $\psi^{\mathrm{NN}^{\prime}}$ changes rapidly, in about $30 \mathrm{fs}$, to a value of $\sim 90^{\circ}$ upon photoexcitation at $t=0$; after the $S_{1} \rightarrow S_{0}$ transition to the ground state, $\psi^{\mathrm{NN}^{\prime}}$ reaches a value of $\sim 180^{\circ}$, thus indicating a successful cis $\rightarrow$ trans isomerisation in both cases. Inspection of the order parameters $\psi^{\mathrm{N}}$ and $\psi^{\mathrm{N}^{\prime}}$ (cf. Fig. 6) reveals that the total change in $\psi^{\mathrm{NN}^{\prime}}$ is due to equal contributions from the two coordination planes at $\mathrm{N}$ and $\mathrm{N}^{\prime}$ in opposite directions. Note that the analysis presented in Fig. 6 shows no significant differences between the liquid and the gas phase. The liquid environment obviously does not impose any major constraints on the dynamics of the CNNC moiety. As discussed above, this is due to the fact that photoisomerisation proceeds through a pedal motion of the CNNC group which does not involve large amplitude rotation of the phenyl rings, as illustrated by the inset of Fig. 6b, which shows the rmsd's of the $\mathrm{N}$ atoms and the phenyl ring centres $\mathrm{R}$. Again, during the first $30 \mathrm{fs}$ it is mainly the translocation of the $\mathrm{N}$ atoms that is responsible for the change in $\psi^{\mathrm{NN}^{\prime}}$ by $\sim 90^{\circ}$, while the phenyl rings remain largely fixed in space.

While the "hula-twist" motion of the CNNC moiety during cis $\rightarrow$ trans photoisomerisation is practically unaffected by the bulk environment, we have observed a pronounced hindrance of the rotation of the phenyl rings about the $\mathrm{C}-\mathrm{N}$ bonds, measured by the rotation angles $\psi^{\mathrm{RN}}$ and $\psi^{\mathrm{R}^{\prime} \mathrm{N}^{\prime}} \cdot{ }^{63}$ In the liquid, relaxation of the molecular structure to the ground state equilibrium following a cis $\rightarrow$ trans switch of the CNNC group is seen to be much slower than in the gas phase.

b. trans $\rightarrow$ cis. Analogous to the above discussion, we now analyze the trans $\rightarrow$ cis photoisomerisation mechanism in the liquid as obtained from nonadiabatic QM/MM simulations. ${ }^{63}$
The time evolution of $\psi^{\mathrm{NN}^{\prime}}$ in the trans $\rightarrow$ cis case is displayed in the bottom panel of Fig. 8b. We observe a much slower decrease in $\psi^{\mathrm{NN}^{\prime}}$ compared to the gas phase (Fig. 8a). For the trajectory shown, a value of $\sim 120^{\circ}$ is reached after $450 \mathrm{fs}$, still far from the $90^{\circ}$ value where the $S_{1} \rightarrow S_{0}$ hop took place in the gas phase. In fact none of the trajectories in the liquid reached $90^{\circ}$, and the average time to reach $120^{\circ}$ is $672 \mathrm{fs}$, compared to $259 \mathrm{fs}$ in the gas phase (see Fig. 7). In contrast to the gas phase, there is no sustained sign-change in either $\psi^{\mathrm{N}}$ or $\psi^{\mathrm{N}^{\prime}}$, explaining the fact that changes in $\psi^{\mathrm{NN}^{\prime}}$ are smaller in the liquid.

It may seem surprising at first that trans $\rightarrow$ cis photoisomerisation is strongly hindered in the bulk, while cis $\rightarrow$ trans is essentially unaffected. This can be easily rationalised, however, in terms of the $S_{1}$ potential landscape (see Section III A 2a, Fig. 5). Vertical excitation of the cis-AB isomer promotes the system to a steep Franck-Condon region in the $S_{1}$, providing a large driving force for isomerisation. The Franck-Condon region for trans-AB, on the other hand, is comparatively flat and the resulting forces are small, rendering trans $\rightarrow$ cis photoisomerisation much more vulnerable to any environmental disturbances. A recent nonadiabatic simulation study of the photoisomerisation of $\mathrm{AB}$ in various organic solvents using a force field derived $a b$ initio by Tiberio et al. ${ }^{131}$ has confirmed the pedal mechanism and the strong impact of the solvent on decay times and quantum yields.

\section{The 8AB8 liquid crystal}

1. Atomistic classical simulations. As described in Section III A 1, we extended and partly reparameterized an existing classical atomistic force field to be able to simulate the liquid crystalline compound 8AB8 which consists of a central azobenzene unit and two octodecane chains connected to the oxygens. We studied the phase behaviour of this compound by classical simulations using replica exchange techniques. ${ }^{46}$ Even though it is possible to use these techniques to equilibrate preset liquid crystalline structures and to draw some conclusions about the stability of certain phases in the atomistic model, the time and length scales required to properly cover liquid crystalline phase transition processes and to extensively investigate the phase behaviour of $8 \mathrm{AB} 8$ can only be reached with a coarse grained simulation approach. Atomistic simulation then again becomes important after a backmapping procedure, to "hand over" well equilibrated structures of the liquid crystalline system obtained from CG simulations to the further classical and QM/MM investigation of the photoisomerization in dense, ordered systems.

2. Coarse grained classical simulations. In ref. 44 we applied a coarse graining scheme that was originally developed for amorphous polymers to liquid crystalline 8AB8. For polymers it is known that the behaviour of the melt is very much determined through chain connectivity and excluded volume interactions of the polymeric beads. Consequently, it is often not essential to introduce attractive (nonbonded/ intermolecular) interactions in order to correctly predict the melt structure and dynamics on the mesoscale. For liquid crystalline $8 \mathrm{AB} 8$ we find that specific (attractive) nonbonded interactions between the different units are required to obtain a CG model that is capable of reproducing the correct liquid crystal behaviour and that is closely linked to the the atomistic 


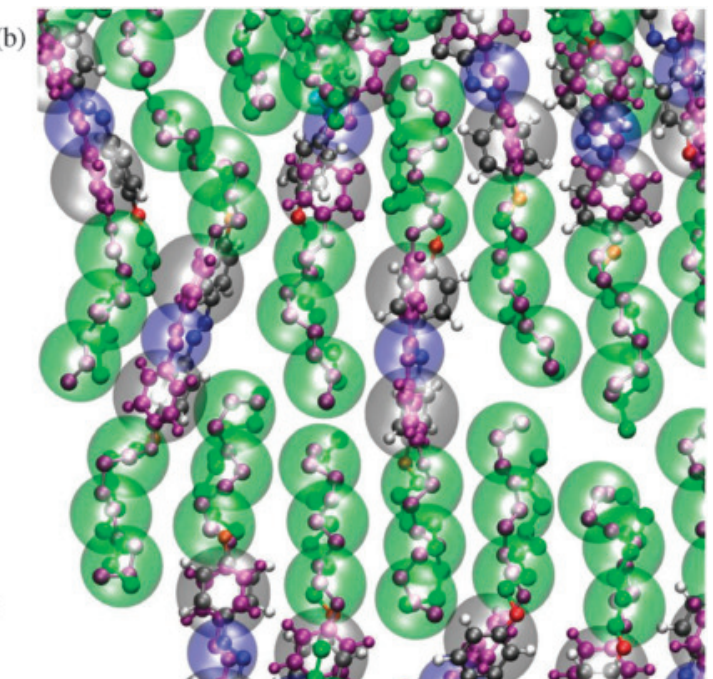

Fig. 11 (left) Chemical structure of (trans) 8AB8 and $\mathrm{MM} \leftrightarrow \mathrm{CG}$ mapping scheme (CG beads are denoted $\mathrm{C}=$ alkyl; $\mathrm{P}=$ phenyl; $\mathrm{N}=$ azo). (right) Snapshot of 8AB8 molecules in a backmapped liquid-crystalline structure. Large spheres: CG beads (green: C; gray: P; blue: N); purple small spheres: re-inserted atomistic coordinates after equilibration with restraining to the CG structure; Green, gray, blue (+ white and red) small spheres: re-inserted atomistic coordinates after 5 ps free MD simulation without restraining potential.

level. Thus it is possible to switch between the levels of resolution. Fig. 11 shows how the atomistic structure of 8AB8 was mapped onto the coarse grained beads. Intramolecular (bonded) CG potentials were obtained from simulations of an all-atom single 8AB8 molecule while intermolecular potentials were developed based on all-atom simulations of isotropic liquids of fragments of the 8AB8 molecule. The isotropic liquids that were used in the parametrization process were liquid benzene, liquid azobenzene (in its trans and in its cis form), liquid octadecane and various mixtures of these compounds. Based on the structure of these liquids (radial distribution functions), nonbonded interaction potentials were determined, both using analytical potential functions and the iterative Boltzmann inversion method.

An overview of all analytical and tabulated interaction functions obtained with this procedure can be found in the Supplementary Material of ref. 44. As an illustration of this structure-based coarse graining method, Fig. 12 shows the radial distribution functions characteristic for liquid trans- and $c i s$-azobenzene. The figure shows the corresponding structure functions from atomistic simulations (mapped onto $\mathrm{CG}$ degrees of freedom) together with the corresponding CG simulations with (numerical) interaction functions obtained from iterative Boltzmann inversion specifically for each isomer. The figure also shows the result of CG simulations with an averaged interaction function which serves as a compromise to be able to use a single set of CG potentials both for trans- and cis-azobenzene. The resulting interaction functions obtained for isotropic liquids were then put together to simulate liquid (trans) 8AB8 to study the liquid crystalline phase behaviour. We found that the use of (soft) analytical potentials which are purely repulsive (in the spirit of the previous coarse graining examples of polymeric systems) did not yield the correct mesophase behaviour of $8 \mathrm{AB} 8$, in fact no long range ordering was observed for the model chosen, even with a rather wide scan of temperatures and pressures. With
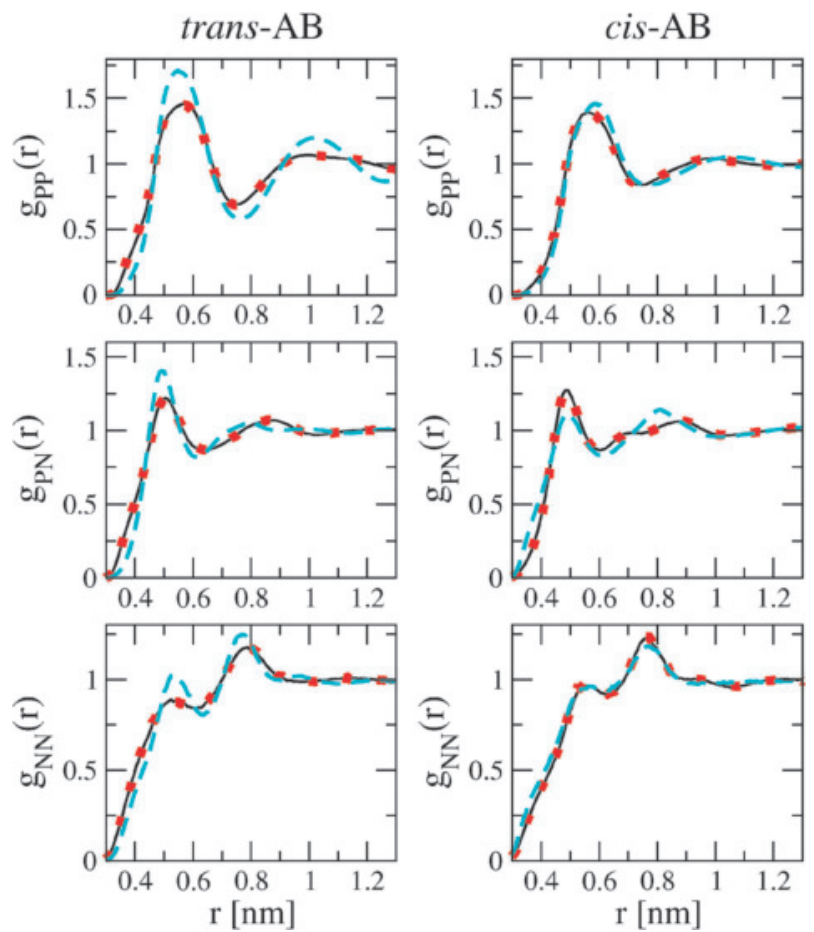

Fig. 12 Radial distribution functions, $g(r)$, for liquid trans-AB (left panels) and cis-AB (right panels). Top panels: phenyl-phenyl $g_{\mathrm{PP}}(r)$. Middle panels: phenyl-azo $g_{\mathrm{PN}}(r)$. Lower panels: azo-azo $g_{\mathrm{NN}}(r)$. Black solid lines: $g(r)$ from atomistic simulation. Red, fat dotted lines: $\mathrm{CG}$ simulation with potentials through iterative Boltzmann inversion specifically for the respective trans and cis compounds. Cyan dashed lines: $\mathrm{CG}$ simulations with average potential as compromise for trans/cis-AB.

potentials generated with the iterative Boltzmann inversion method, i.e. numerical (tabulated) potentials which are also partly attractive, it is however possible to observe liquid crystalline structures. Thus, for the given molecule, i.e. the 

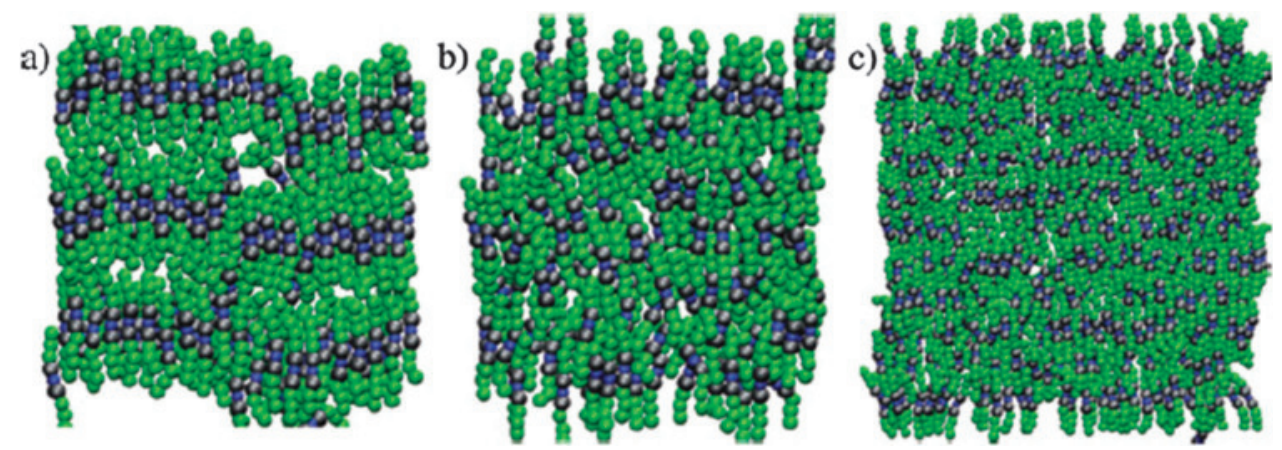

Fig. 13 Snapshots of slices through the liquid crystal system from simulations at $T=0.8 T_{0}\left(T_{0}=400 \mathrm{~K}\right)$ with the average- $8 \mathrm{AB} 8 \mathrm{FF}$. (a) 1323 $8 \mathrm{AB} 8$ molecules, density $=1.44$ molecules per $\mathrm{nm}^{3}$; (b) $13238 \mathrm{AB} 8$ molecules, density $=1.62$ molecules per nm ${ }^{3}$; (c) $61258 \mathrm{AB} 8 \mathrm{molecules}$, density $=1.52$ molecules per $\mathrm{nm}^{3}$.

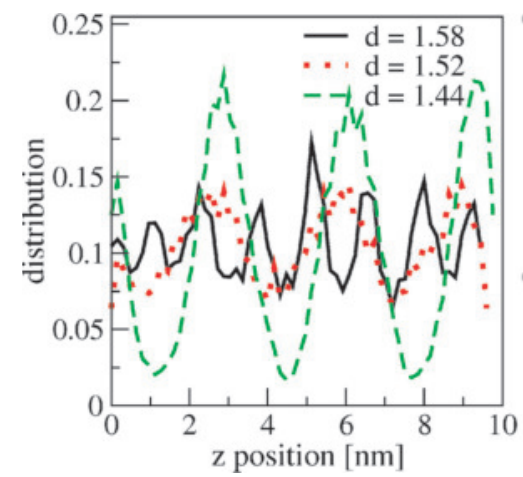

Fig. 14 Distribution of centres of 8AB8 molecules ( $\mathrm{N}$ beads) along the $z$-direction of the simulation box of the liquid crystal system at $T=0.8 T_{0}\left(T_{0}=400 \mathrm{~K}\right)$ and various densities (see legend, densities are in molecules per $\mathrm{nm}^{3}$ ).

given size and shape of the mesogen and the given molecular flexibility of the alkoxy tails, it seems to be important to account for attractions between the different beads in the $\mathrm{CG}$ model in order to reproduce liquid crystalline phases of 8AB8. By varying simulation temperature and density it was possible to distinguish structures with smectic layers and more disordered structures, which are however not truly nematic but still exhibit a varying degree of positional order with partly interdigitated smectic layers, as illustrated in Fig. 14 which shows the $z$-positional order in the liquid crystal systems. Several snapshots of the corresponding 8AB8 liquid crystal simulations at different densities and system sizes can be seen in Fig. 13. At this point it should be noted that with this approach of building a CG model on an atomistic force field description of the molecule, possible weaknesses of the atomistic model will be automatically transfered to the CG model. With the given approach the mesoscale simulations maintain an important link to the chemical structure, and through the inverse mapping procedure it is possible to obtain atomistic coordinates of the system as illustrated in Fig. 11. This is important for passing down the $\mathrm{CG}$ configuration and velocities to the MM level below, which, in turn, serves as a starting point for a na-QM/MM simulation during which one of the $8 \mathrm{AB} 8$ molecules is photoswitched from trans to cis.

\section{Summary and perspective}

We have presented a multiscale simulation approach which links nonadiabatic ab initio molecular dynamics, atomistic classical molecular dynamics, and coarse grained classical molecular dynamics, to make possible the simulation of mesoscopic processes triggered by quantum-mechanical events highly local in space and time. As a test bed for our combined approach we chose the liquid crystalline system 8AB8, consisting of an azobenzene chromophore embedded in a hydrocarbon chain. By switching a fraction of $\mathrm{AB}$ units from trans- $\mathrm{AB}$ to cis-AB using light of a suitable wavelength, transitions between ordered and disordered phases can be induced in the 8AB8 liquid crystal.

First, an atomistic force field for $\mathrm{AB}$ and $\mathrm{AB}$ chain derivatives was derived from AIMD data in the gas phase, and was then shown to be suitable for bulk liquid $\mathrm{AB}$ and liquid crystalline 8 AB8 as well. The atomistic force field was the prerequisite to be able to perform nonadiabatic QM/MM simulations of the photoisomerisation of $\mathrm{AB}$ in the bulk, and it formed the basis for the development of a coarse grained representation of $8 \mathrm{AB} 8$, which was required to study the phase behaviour of the liquid crystal. We have indeed demonstrated that the $\mathrm{CG}$ force field is capable of reproducing several ordered phases of the $8 \mathrm{AB} 8$ liquid crystal and phase transitions between them.

We have gained unprecedented insights into the photoisomerisation mechanism of $\mathrm{AB}$ from na-QM simulations. A clear relationship between the molecular structure of a particular AB-based photoswitch and its relevant properties, such as photoisomerisation efficiency, has been established. This could pave the way for the rational design of improved photoswitches for light-controllable nano-devices and materials. Nonadiabatic QM/MM simulations of the photoisomerisation in liquid $\mathrm{AB}$ have revealed that the cis $\rightarrow$ trans reaction is largely unaffected by the environment, while trans $\rightarrow$ cis is strongly hindered in the bulk. The respective behaviours of cis-AB and trans-AB could be traced back to their photoisomerisation mechanisms and rationalised in terms of the potential energy landscape.

All the necessary parts to perform a na-QM/MM/CG multiscale simulation are now available and can be applied to study photoisomerisation in $8 \mathrm{AB} 8$ and its effect on the 
liquid crystalline order. The most straightforward way of doing this is in a sequential fashion. Having built the multiscale model from the bottom up, i.e. based on first principles, the best way to tackle the liquid crystal is to apply the individual tools at the different scales in a cyclic manner, starting from the top. This means that first an ordered liquid crystalline phase needs to be produced in a $\mathrm{CG}$ simulation. The CG system then has to be back-mapped onto the atomistic representation using the procedure presented here and an MM simulation needs to be performed to reequilibrate the system. To simulate a photoisomerisation event, an na-QM/MM simulation has to be carried out subsequently. From this bottom layer, the information is then passed back to the $\mathrm{MM}$ and eventually to the $\mathrm{CG}$ level to complete the cycle.

Since, to induce a phase transition in $8 \mathrm{AB} 8$, a significant fraction of molecules need to be switched, it is desirable to develop a purely analytical switching potential based on the knowledge gained from na-QM/MM simulations about the mechanism. Such a switching potential is currently being designed and tested.

At present, the multiscale simulations involve different codes for the different layers. However, we aim to implement a simultaneous na-QM/MM/CG simulation method, which would facilitate applications to very extended systems, albeit at the expense of a limited timescale. This means that all the methods outlined in this Perspective can be potentially combined in a single software package. Therefore, parts of the multiscale strategy reviewed here, such as the adaptive resolution scheme, are currently being extended to be able to treat concurrently in a robust way many scales, including the quantum level, in a single code.

Other desirable extensions of the current method are, for instance, an adaptive QM/MM partitioning, beyond the path integral representation of atomic nuclei (without treating electrons explicitly), allowing for particle exchange between the $\mathrm{QM}$ and the MM regions, multiple $\mathrm{QM}$ regions, and a theoretically sound method of switching "on" and "off" a QM region. These are issues that need to be solved not only in the context of the current project, but also for hybrid simulation schemes in general.

The multiscale strategy and methodology developed here presents a powerful tool, first and foremost in the ever growing field of light-addressable azo-materials, but at the same time it is transferable to a plethora of other applications, including (photo)biochemistry and -biophysics.

\section{Acknowledgements}

We are grateful to the Volkswagen Stiftung for supporting our project "Adaptive Multiscale Simulation: Connecting the Quantum to the Mesoscopic Level" within the framework of the program "New Conceptual Approaches to Modeling and Simulation of Complex Systems - Computer Simulation of Molecular and Cellular Biosystems as well as Complex Soft Matter'. The simulations were performed using resources from NIC Jülich, Bovilab@RUB and Rechnerverbund-NRW.

\section{References}

1 R. Car and M. Parrinello, Phys. Rev. Lett., 1985, 55, 2471.

2 D. Marx and J. Hutter, in Modern Methods and Algorithms of Quantum Chemistry, ed. J. Grotendorst, NIC, Jülich, 2000, www.theochem.rub.de/go/cprev.html, accessed August 2010.

3 D. Marx and J. Hutter, Ab initio Molecular Dynamics: Basic Theory and Advanced Methods, Cambridge University Press, Cambridge, 2009.

4 J. Aqvis and A. Warshel, Chem. Rev., 1993, 93, 2523.

5 J. L. Gao and D. G. Truhlar, Annu. Rev. Phys. Chem., 2002, 53, 467.

6 P. Carloni, U. Rothlisberger and M. Parrinello, Acc. Chem. Res., 2002, 35, 455

7 P. Sherwood, A. H. de Vries, M. F. Guest, G. Schreckenbach, C. R. A. Catlow, S. A. French, A. A. Sokol, S. T. Bromley, W. Thiel and A. J. Turner, et al., J. Mol. Struct. (THEOCHEM), 2003, 632, 1 .

8 G. Tresadern, P. F. Faulder, M. P. Gleeson, Z. Tai, G. MacKenzie, N. A. Burton and I. H. Hillier, Theor. Chem. Acc., 2003, 109, 108.

9 H. M. Senn and W. Thiel, Angew. Chem., Int. Ed., 2009, 48, 1198.

10 Coarse-Graining of Condensed Phase and Biomolecular Systems, ed. G. A. Voth, Chapman and Hall/CRC Press, Taylor and Francis Group, 2009.

11 M. L. Klein and W. Shinoda, Science, 2008, 321, 798.

12 J. Baschnagel, K. Binder, P. Doruker, A. A. Gusev, O. Hahn, K. Kremer, W. L. Mattice, F. Muller-Plathe, M. Murat and W. Paul, et al., Adv. Polym. Sci., 2000, 152, 41.

13 W. Tschöp, K. Kremer, J. Batoulis, T. Burger and O. Hahn, Acta Polym., 1998, 49, 61.

14 C. F. Abrams and K. Kremer, Macromolecules, 2003, 36, 260.

15 V. A. Harmandaris, N. P. Adhikari, N. F. A. van der Vegt and K. Kremer, Macromolecules, 2006, 39, 6708.

16 Q. Sun and R. Faller, J. Chem. Theory Comput., 2006, 2, 607.

17 T. Spyriouni, C. Tzoumanekas, D. Theodorou, F. Mueller-Plathe and G. Milano, Macromolecules, 2007, 40, 3876.

18 V. A. Harmandaris, D. Reith, N. F. A. van der Vegt and K. Kremer, Macromol. Chem. Phys., 2007, 208, 2109.

19 V. A. Harmandaris and K. Kremer, Macromolecules, 2009, 42, 791.

20 D. Fritz, V. A. Harmandaris, K. Kremer and N. F. A. van der Vegt, Macromolecules, 2009, 42, 7579.

21 M. Muller, K. Katsov and M. Schick, Phys. Rep., 2006, 434, 113.

22 S. J. Marrink, H. J. Risselada, S. Yefimov, D. P. Tieleman and A. H. de Vries, J. Phys. Chem. B, 2007, 111, 7812.

23 B. J. Reynwar, G. Illya, V. A. Harmandaris, M. M. Müller, K. Kremer and M. Deserno, Nature, 2007, 447, 461.

24 M. Deserno, Macromol. Rapid Commun., 2009, 30, 752.

25 H. D. Nguyen and C. K. Hall, Proc. Natl. Acad. Sci. U. S. A., 2004, 101, 16180.

26 G. Bellesia and J. E. Shea, J. Chem. Phys., 2007, 126, 245104.

27 I. F. Thorpe, J. Zhou and G. A. Voth, J. Phys. Chem. B, 2008, 112, 13079.

28 A. Villa, C. Peter and N. F. A. van der Vegt, Phys. Chem. Chem. Phys., 2009, 11, 2077.

29 A. Villa, N. F. A. van der Vegt and C. Peter, Phys. Chem. Chem. Phys., 2009, 11, 2068.

30 T. Head-Gordon and S. Brown, Curr. Opin. Struct. Biol., 2003, 13, 160 .

31 C. Clementi, Curr. Opin. Struct. Biol., 2008, 18, 10.

32 V. Tozzini, Acc. Chem. Res., 2010, 43, 220.

33 C. Peter and K. Kremer, Faraday Discuss., 2010, 144, 9.

34 A. P. Lyubartsev and A. Laaksonen, Phys. Rev. E: Stat. Phys., Plasmas, Fluids, Relat. Interdiscip. Top., 1995, 52, 3730.

35 F. Müller-Plathe, ChemPhysChem, 2002, 3, 754.

36 M. E. Johnson, T. Head-Gordon and A. A. Louis, J. Chem. Phys., 2007, 126, 144509.

37 L. Monticelli, S. K. Kandasamy, X. Periole, R. G. Larson, D. P. Tieleman and S. J. Marrink, J. Chem. Theory Comput., 2008, 4, 819 .

38 W. Noid, J. Chu, G. Ayton and G. Voth, J. Phys. Chem. B, 2007, 111, 4116.

39 W. Mullinax and J. W. Noid, Phys. Rev. Lett., 2009, 103, 198104. 
40 H. Wang, C. Junghans and K. Kremer, Eur. Phys. J. E, 2009, 28, 221

41 A. Lyubartsev, A. Mirzoev, L. Chen and A. Laaksonen, Faraday Discuss., 2010, 144, 43.

42 B. Hess, S. Leon, N. van der Vegt and K. Kremer, Soft Matter, 2006, 2, 409.

43 G. Santangelo, A. Di Matteo, F. Muller-Plathe and G. Milano, J. Phys. Chem. B, 2007, 111, 2765.

44 C. Peter, L. Delle Site and K. Kremer, Soft Matter, 2008, 4, 859.

45 X. Chen, P. Carbone, G. Santangelo, A. Di Matteo, G. Milano and F. Muller-Plathe, Phys. Chem. Chem. Phys., 2009, 11, 1977.

46 M. Böckmann, C. Peter, L. Delle Site, N. L. Doltsinis, K. Kremer and D. Marx, J. Chem. Theory Comput., 2007, 3, 1789.

47 G. S. Kumar and D. C. Neckers, Chem. Rev., 1989, 89, 1915.

48 T. Ikeda and O. Tsutsumi, Science, 1995, 268, 1873.

49 Molecular Switches, ed. B. L. Feringa, Wiley-VCH, Weinheim, 2001.

50 Photoreactive organic thin films, ed. Z. Sekkat and W. Knoll, Academic Press, San Diego, 2002.

51 A. Natansohn and P. Rochon, Chem. Rev., 2002, 102, 4139.

52 A. Stolow, Annu. Rev. Phys. Chem., 2003, 54, 89.

53 S. Spörlein, H. Carstens, H. Satzger, C. Renner, R. Behrendt, L. Moroder, P. Tavan, W. Zinth and J. Wachtveitl, Proc. Natl. Acad. Sci. U. S. A., 2002, 99, 7998.

54 Y. Yu, M. Nakano and T. Ikeda, Nature, 2003, 425, 145.

55 I. Banerjee, L. Yu and H. Matsui, J. Am. Chem. Soc., 2003, 125, 9542.

56 W. R. Browne and B. L. Feringa, Nat. Nanotechnol., 2006, 1, 25.

57 T. Hugel, N. B. Holland, A. Cattani, L. Moroder, M. Seitz and H. E. Gaub, Science, 2002, 296, 1103.

58 A. Khan, C. Kaiser and S. Hecht, Angew. Chem., Int. Ed., 2006, 45, 1878.

59 N. L. Doltsinis and D. Marx, Phys. Rev. Lett., 2002, 88, 166402.

60 N. L. Doltsinis and D. Marx, J. Theor. Comput. Chem., 2002, 1, 319.

61 A. Laio, J. VandeVondele and U. Rothlisberger, J. Chem. Phys., 2002, 116, 6941.

62 M. Böckmann, N. L. Doltsinis and D. Marx, Phys. Rev. E: Stat., Nonlinear, Soft Matter Phys., 2008, 78, 036101.

63 M. Böckmann, N. L. Doltsinis and D. Marx, J. Phys. Chem. A, 2010, 114, 745 .

64 T. Kerdcharoen, K. R. Liedl and B. M. Rode, Chem. Phys., 1996, 211, 313 .

65 T. S. Hofer, A. B. Pribil, B. R. Randolf and B. M. Rode, J. Am. Chem. Soc., 2005, 127, 14231.

66 T. Kerdcharoen and K. Morokuma, Chem. Phys. Lett., 2002, 355, 257.

67 R. Bulo, B. Ensing, J. Sikkema and L. Visscher, J. Chem. Theory Comput., 2009, 5, 2212.

68 A. Heyden and D. G. Truhlar, J. Chem. Theory Comput., 2008, 4, 217

69 G. Csanyi, T. Albaret, M. C. Payne and A. D. Vita, Phys. Rev. Lett., 2004, 93, 175503.

70 A. B. Poma and L. Delle Site, Phys. Rev. Lett., 2010, 104, 250201.

71 M. Praprotnik, L. Delle Site and K. Kremer, J. Chem. Phys., 2005, 123, 224106.

72 M. Praprotnik, L. Delle Site and K. Kremer, Phys. Rev. E: Stat. Nonlinear, Soft Matter Phys., 2006, 73, 066701.

73 M. Praprotnik, K. Kremer and L. Delle Site, Phys. Rev. E: Stat., Nonlinear, Soft Matter Phys., 2007, 75, 017701a.

74 M. Praprotnik, K. Kremer and L. Delle Site, J. Phys. A: Math. Theor., 2007, 40, 017701b.

75 R. G. Parr and W. Yang, Density Functional Theory of Atoms and Molecules, Oxford University Press, Oxford, 1989.

76 R. M. Dreizler and E. K. U. Gross, Density Functional Theory, Springer, Berlin, 1990.

77 N. L. Doltsinis, in Quantum Simulations of Complex Many-Body Systems: From Theory to Algorithms, ed. J. Grotendorst, D. Marx and A. Muramatsu, NIC, FZ Jülich, 2002, www.fz-juelich.de/ nic-series/volume10/doltsinis.pdf.

78 N. L. Doltsinis, in Computational Nanoscience Do it Yourself! ed. J. Grotendorst, S. Blügel and D. Marx, NIC, FZ Jülich, 2006, www.fz-juelich.de/nic-series/volume31/doltsinis1.pdf.

79 I. Frank, J. Hutter, D. Marx and M. Parrinello, J. Chem. Phys., 1998, 108, 4060.
80 S. Grimm, C. Nonnenberg and I. Frank, J. Chem. Phys., 2003, 119, 11574 .

81 S. Grimm, C. Nonnenberg and I. Frank, J. Chem. Phys., 2003, 119, 11585

82 J. C. Tully, J. Chem. Phys., 1990, 93, 1061.

83 U. Müller and G. Stock, J. Chem. Phys., 1997, 107, 6230.

84 S. Grimm, C. Nonnenberg and I. Frank, J. Chem. Phys., 2003, 119, 11574; S. Grimm, C. Nonnenberg and I. Frank, J. Chem. Phys., 2003, 119, 11585.

85 N. L. Doltsinis, Mol. Phys., 2004, 102, 499.

86 H. Langer and N. L. Doltsinis, Phys. Chem. Chem. Phys., 2004, 6, 2742.

87 H. Langer, N. L. Doltsinis and D. Marx, ChemPhysChem, 2005, 6, 1734.

88 P. R. L. Markwick and N. L. Doltsinis, J. Chem. Phys., 2007, 126, 175102

89 N. L. Doltsinis, P. R. L. Markwick, H. Nieber and H. Langer, in Radiation Induced Molecular Phenomena in Nucleic Acid, ed. M. K. Shukla and J. Leszczynski, Springer, Netherlands, 2008.

90 H. Nieber and N. L. Doltsinis, Chem. Phys., 2008, 347, 405.

91 S. R. Billeter and D. Egli, J. Chem. Phys., 2006, 125, 224103.

92 W. F. van Gunsteren and H. J. C. Berendsen, BIOMOS B.V., Zürich/Groningen, 1996.

93 R. Baron, D. Trzesniak, A. H. de Vries, A. Elsener, S. J. Marrink and W. F. van Gunsteren, ChemPhysChem, 2007, 8, 452.

94 V. Ruehle, C. Junghans, A. Lukyanov, K. Kremer and D. Andrienko, J. Chem. Theory Comput., 2009, 5, 3211.

95 F. Ercolessi and J. B. Adams, Europhys. Lett., 1994, 26, 583.

96 S. Izvekov and G. A. Voth, J. Phys. Chem. B, 2005, 109, 2469.

97 G. S. Ayton, W. G. Noid and G. A. Voth, Curr. Opin. Struct. Biol., 2007, 17, 192.

98 A. Villa, C. Peter and N. F. A. van der Vegt, J. Chem. Theory Comput., 2010, 6, 2434.

99 A. Chaimovich and M. S. Shell, Phys. Chem. Chem. Phys., 2009, 11, 1901.

100 J. R. Silbermann, S. H. L. Klapp, M. Schoen, N. Chennamsetty, H. Bock and K. E. Gubbins, J. Chem. Phys., 2006, 124, 074105.

101 D. Reith, M. Putz and F. Muller-Plathe, J. Comput. Chem., 2003, 24, 1624.

102 M. Praprotnik, L. Delle Site and K. Kremer, Annu. Rev. Phys. Chem., 2008, 59, 545.

103 B. Ensing, S. O. Nielsen, P. B. Moore, M. L. Klein and M. Parrinello, J. Chem. Theory Comput., 2007, 3, 1100.

104 S. Izvekov and G. A. Voth, J. Chem. Theory Comput., 2009, 5, 3232.

105 S. Poblete, M. Praprotnik, K. Kremer and L. Delle Site, J. Chem. Phys., 2010, 132, 114101, ISSN 00219606.

106 L. Delle Site, Phys. Rev. E: Stat., Nonlinear, Soft Matter Phys., 2007, 76, 047701

107 M. Praprotnik, L. Delle Site and K. Kremer, J. Chem. Phys., 2007, 126, 134902.

108 S. Matysiak, M. Praprotnik, L. Delle Site, K. Kremer and C. Clementi, J. Phys.: Condens. Matter, 2007, 19, 292201.

109 S. Matysiak, C. Clementi, M. Praprotnik, K. Kremer and L. Delle Site, J. Chem. Phys., 2008, 128, 024503.

110 R. Delgado-Buscalioni, K. Kremer and M. Praprotnik, J. Chem. Phys., 2008, 128, 114110.

111 R. Delgado-Buscalioni, K. Kremer and M. Praprotnik, J. Chem. Phys., 2009, 131, 244107.

112 B. Lambeth, C. Junghans, K. Kremer, C. Clementi and L. Delle Site, J. Chem. Phys., 2010, 133, 221101.

113 C. I. Bayly, P. Cieplak, W. Cornell and P. A. Kollman, J. Phys. Chem., 1993, 97, 10269

114 D. A. Case, D. A. Pearlman, J. W. Caldwell, T. E. Cheatham III, J. Wang, W. S. Ross, C. L. Simmerling, T. A. Darden, K. M. Merz, R. V. Stanton, A. L. Cheng, J. J. Vincent, M. Crowley, V. Tsui, H. Gohlke, R. J. Radmer, Y. Duan, J. Pitera, I. Massova, G. L. Seibel, U. C. Singh, P. K. Weiner and P. A. Kollman, AMBER 7, University of California, San Francisco, 2002.

115 A. Cembran, F. Bernardi, M. Garavelli, L. Gagliardi and G. Orlandi, J. Am. Chem. Soc., 2004, 126, 3234.

116 A. W. Adamson, A. Vogler, H. Kunkely and R. Wachter, J. Am. Chem. Soc., 1978, 100, 1300. 
117 J. Harada, K. Ogawa and S. Tomoda, Acta Crystallogr., Sect. B: Struct. Sci., 1997, 53, 662.

118 T. Pancur, F. Renth, F. Temps, B. Harbaum, A. Krüger, R. Herges and C. Näther, Phys. Chem. Chem. Phys., 2005, 7, 1985.

119 C. M. Stuart, R. R. Frontiera and R. A. Mathies, J. Phys. Chem. A, 2007, 111, 12072.

120 C.-W. Chang, Y.-C. Lu, T.-T. Wang and E. W.-G. Diau, J. Am Chem. Soc., 2004, 126, 10109.

121 H. Satzger, S. Spörlein, J. Wachtveitl, W. Zinth and P. Gilch, Chem. Phys. Lett., 2003, 372, 216.

122 R. Siewertsen, H. Neumann, B. Buchheim-Stehn, R. Herges, C. Näther, F. Renth and F. Temps, J. Am. Chem. Soc., 2009, 131, 15594.

123 M. Böckmann, N. L. Doltsinis and D. Marx, Angew. Chem., Int. Ed., 2010, 49, 3382.
124 H. Flyvbjerg and H. G. Petersen, J. Chem. Phys., 1989, 91, 461.

125 A. W. Jasper, S. Nangia, C. Zhu and D. G. Truhlar, Acc. Chem. Res., 2006, 39, 101.

126 M. D. Hack, A. M. Wensmann, D. G. Truhlar, M. Ben-Nun and T. J. Martnez, J. Chem. Phys., 2001, 115, 1172.

127 A. Toniolo, C. Ciminelli, M. Persico and T. Martinez, J. Chem. Phys., 2005, 123, 234308.

128 R. Turanský, M. Konôpka, N. L. Doltsinis, I. Štich and D. Marx, ChemPhysChem, 2010, 11, 345.

129 R. Turanský, M. Konôpka, N. L. Doltsinis, I. Štich and D. Marx, Phys. Chem. Chem. Phys., 2010, 12, 13922.

130 O. Carstensen, J. Sielk, J. B. Schönborn, G. Granucci and B. Hartke, J. Chem. Phys., 2010, 133, 124305.

131 G. Tiberio, L. Muccioli, R. Berardi and C. Zannoni, ChemPhysChem, 2010, 11, 1018. 